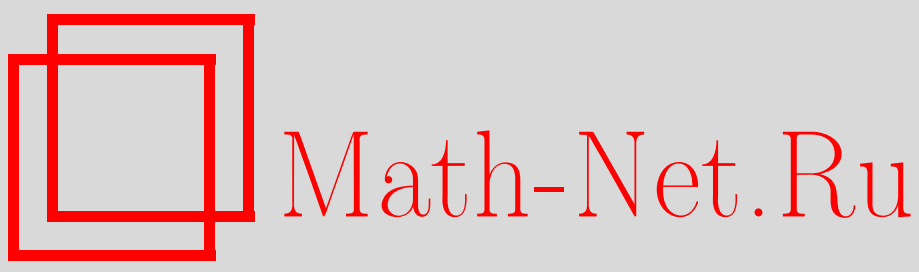

Ю. Л. Ершов, Абстрактная теория полей классов (финитарный подход), Матем. сб., 2003, том 194, номер 2, 37-60

DOI: https://doi.org/10.4213/sm712

Использование Общероссийского математического портала Math-Net.Ru подразумевает, что вы прочитали и согласны с пользовательским соглашением http://www . mathnet.ru/rus/agreement

Параметры загрузки:

IP: 52.23 .180 .231

26 апреля 2023 г., 12:32:59 
УДК $510.53+512.52$

\author{
Ю. Л. Ершов
}

\title{
Абстрактная теория полей классов (финитарный подход)
}

\begin{abstract}
В работе дано определение гомоморфизма взаимности в абстрактной теории полей классов Нейкирха, используюее лишь достаточно большие, но конечные дополнительные неразветвленные расширения. Это позволит применить построенную теорию для эффективизации (алгоритмизации) как локальной, так и глобальной теории полей классов.

Комбинация подходов Нейкирха и Хазевинкеля, использованная в этой статье, проливает дополнительньй свет на теорию полей классов даже на абстрактном уровне ее изложения.

Библиографоя: 5 названий.
\end{abstract}

Ю. Нейкирх в [1], [2] сумел выделить общую часть теории полей классов (как локальной, так и глобальной) как фрагмент теории когомологий проконечных групा в размерностях $-1,0$ при выполнении ряда естественных аксиом. В его изложении для определения гомоморфизма взаимности используются максимальные неразветвленные расширения, являюшиеся бесконечными расширениями. В настоящей работе предложен подход, основанный на подходе Нейкирха, но используюший только конечные расширения. Это позволит применять построенную теорию для эффективизации (алгоритмизации) как локальной, так и глобальной теории полей классов.

В предлагаемом изложении используется также и подход Хазевинкеля к определению (локальной) теории полей классов, описанной в книге Ивасавы [3]. Комбинация этих подходов проливает дополнительный свет на теорию полей классов даже на абстрактном уровне ее изложения.

Пусть $G$-проконечная группа; будем предполагать, что существует поле $k$, его расширение Галуа $\bar{k}$ такое, что $G \simeq G(\bar{k} / k)$ (по теореме Уотерхауза [4] любая проконечная группа может быть так "реализована"). Тогда по теории Галуа существует естественное взаимно однозначное соответствие между всеми промежуточными полями $K, k \leqslant K \leqslant \bar{k}$, и замкнутыми подгруппами группы $G: K \longmapsto G_{K} \rightleftharpoons$ $\left\{\sigma \mid \sigma \in G, a^{\sigma}=a\right.$ для всех $\left.a \in K\right\} ; G_{k}=G, G_{\bar{k}}=\langle 1\rangle$.

Заметим, что вложение $G_{L} \leqslant G_{K}$ соответствует вложению $K \leqslant L ; L(\geqslant K)$ - расширение Галуа поля $K$ тогда и только тогда, когда $G_{L}$ - нормальная подгруппа $G_{K}$ (и тогда группа Галуа $G(L / K)$ этого расширения есть $G_{K} / G_{L}$ ); если $\sigma \in G_{K}$, то полю $L^{\sigma} \rightleftharpoons\left\{a \mid a \in L, a^{\sigma}=a\right\}$ соответствует подгруппа $\overline{\left\langle G_{L}, \sigma\right\rangle}$ (замькание подгруппы $\left.\left\langle G_{L}, \sigma\right\rangle\right)$. Заметим, что это обозначение $L^{\sigma}$ не согласуется с обозначением $L^{\sigma}$ в [2].

Для $\sigma \in G$ поле, соответствуюшее подгруппе $\sigma^{-1} G_{L} \sigma$, будем обозначать через $\sigma(L)$ (в [2] это поле обозначается как $L^{\sigma}$ ). 
Если $M \geqslant L(\geqslant K)$ - расширения Галуа поля $K, \sigma \in G(M / K)$, то через $\sigma \uparrow L$ будем обозначать образ элемента $\sigma$ в групше $G(L / K)$ при естественном эпиморфизме $G(M / K)\left(=G_{K} / G_{M}\right) \longrightarrow G(L / K)\left(=G_{K} / G_{L}\right)\left(L \leqslant M \Longrightarrow G_{M} \leqslant G_{L}\right)$.

Пусть $d: G \longrightarrow \widehat{\mathbb{Z}}$ - некоторый фиксированный эпиморфизм проконечных групп; через $I$ обозначим ядро этого эпиморфизма; $I=G_{\widetilde{k}}$ для подходяшего поля $\widetilde{k}$ $(k \leqslant \widetilde{k} \leqslant \bar{k})$. Для произвольного промежуточного поля $K$ группа $I_{K} \rightleftharpoons G_{K} \cap I$ называется группой инериии поля $K$. Пусть $f_{K} \rightleftharpoons\left[\widehat{\mathbb{Z}}: d\left(G_{K}\right)\right], e_{K} \rightleftharpoons\left[I: I_{K}\right]$. Если $f_{K}$ конечно (например, в случае, когда $K$ - конечное расширение $k$ ), то определен эпиморфизм

$$
d_{K} \rightleftharpoons f_{K}^{-1} d: G_{K} \longmapsto \widehat{\mathbb{Z}}
$$

Для расширения $K \leqslant L$ степень инерции $f_{L / K}$ расширения определена как $\left[d\left(G_{K}\right): d\left(G_{L}\right)\right]$, а индекс ветвления $e_{L / K}-$ как $\left[I_{K}: I_{L}\right]$.

Для башни расширений $K \leqslant L \leqslant M$ имеются очевидные соотношения $f_{M / K}=$ $f_{L / K} \cdot f_{M / L}$ и $e_{M / K}=e_{L / K} \cdot e_{M / L}$.

ПРЕДЛОЖЕНИЕ 1 [2; предложение (4.2)]. Для любого расширения $K \leqslant L$ справедливо основное равенство $[L: K]=f_{L / K} \cdot e_{L / K}$.

Расширение $K \leqslant L$ называется неразветвленным, если $e_{L / K}=1$; вполне разветвленным, если $f_{L / K}=1$.

ЗАмЕчАнИЕ 1 . Всякое неразветвленное расширение $K \leqslant L$ является абелевым расширением Галуа (циклическим, если $[L: K]<\omega)$.

Действительно, если $e_{L / K}=1$, то $I_{K}=I_{L} ; G_{L} / I_{L} \leqslant G_{K} / I_{K} \simeq d\left(G_{K}\right) \leqslant \widehat{\mathbb{Z}}$. Следовательно, $G_{L} / I_{L}$ нормальна в $G_{K} / I_{K}, G_{L}$ нормальна в $G_{K}$ и $G(L / K)=$ $G_{K} / G_{L} \simeq d\left(G_{K}\right) / d\left(G_{L}\right)$.

ЗАмЕЧАнИЕ 2. Для любого расширения $K \leqslant L$ сушествует наибольшее подрасширение $L_{u}^{K}$ такое, что $K \leqslant L_{u}^{K} \leqslant L, L_{u}^{K} \geqslant K$ - неразветвленное, а $L \geqslant L_{u}^{K}-$ вполне разветвленное расширение.

Поле $L_{u}^{K}$ соответствует замкнутой подгруппе $G_{L} I_{K}\left(G_{L} \leqslant G_{L} I_{K} \leqslant G_{K}\right)$. Действительно, легко видеть из определения, что $I_{L_{u}^{K}}=I_{K}\left(I_{L_{u}^{K}}=G_{L} I_{K} \cap I \geqslant I_{K}\right.$; $\left.I_{L_{u}^{K}}=G_{L} I_{K} \cap I \leqslant G_{K} \cap I=I_{K}\right)$. Следовательно, $L_{u}^{K} \geqslant K$ - неразветвленное расширение. Так как $I_{K} \leqslant I=\operatorname{Ker} d$, то $d\left(G_{L}\right)=d\left(G_{L} I_{K}\right)=d\left(G_{L_{u}^{K}}\right)$, следовательно, $f_{L / L_{u}^{K}}=1$, т.е. $L \geqslant L_{u}^{K}$ - вполне разветвленное расширение.

Для $K \geqslant k$ пусть $\mathbb{Z}_{K} \rightleftharpoons \widehat{\mathbb{Z}} / d\left(G_{K}\right)$, и если $K$ - расширение Галуа поля $k$, то сушествует естественньй эпиморфизм $d^{K}: G(K / k) \longrightarrow \mathbb{Z}_{K}$, индуцированньй эпиморфизмом $d$.

Для $K \leqslant L$ пусть $\mathbb{Z}_{L / K} \rightleftharpoons \widehat{\mathbb{Z}} / d_{K}\left(G_{L}\right)$, и если $f_{K}<\omega$ и $L-$ расширение Галуа поля $K$, то существует естественный эпиморфизм $d_{K}^{L}: G(L / K) \longrightarrow \mathbb{Z}_{L / K}$, индуцированный эпиморфизмом $d_{K}$. Ядро эпиморфиизма $d_{K}^{L}$ обозначим через $I_{K}^{L}$. Заметим, что группа $\mathbb{Z}_{L / K}$ естественно изоморфна групе $G\left(L_{u}^{K} / K\right)$ и $\left|\mathbb{Z}_{L / K}\right|=$ $f_{L / K}$.

Если $K \leqslant L \leqslant M, f_{K}<\omega, L$ и $M-$ расшшиения Галуа поля $K$, то следующая 
диаграмма

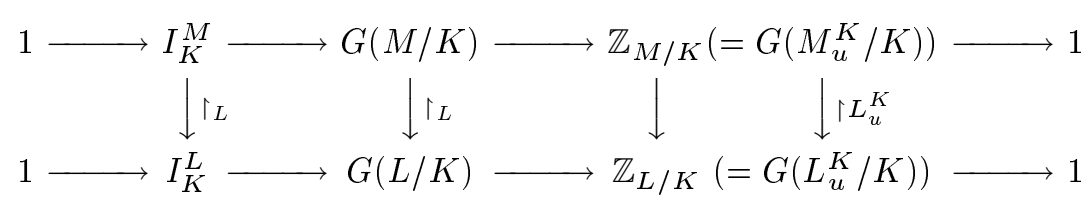

коммутативна, где вертикальные эпиморфизмы естественно определены $\left(G_{M} \leqslant\right.$ $\left.G_{L}\right)$.

ЗАмечАнИЕ 3 . Если $L$ - расширение Галуа поля $K, M$ - неразветвленное расширение $L$, то $M$ является расширением Галуа поля $K$.

Действительно, рассмотрение диаграммы, изображенной на рис. 1, показывает, что в случае неразветвленности расширения $M \geqslant L$ имеет место соотношение $M=L M_{u}^{K}=L \otimes_{L_{u}^{K}} M_{u}^{K}$; т.е. $M$ является композитом расширений Галуа $K$ и $M_{u}^{K}$ поля $K$.

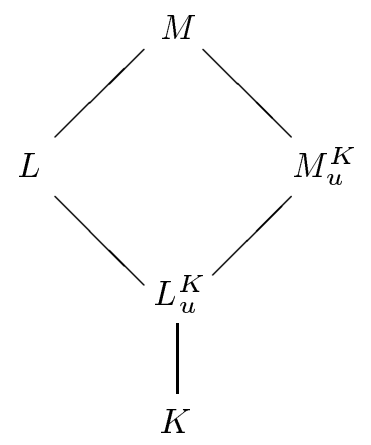

Рис. 1

ПРЕДЛОЖЕНИЕ 2. Для любого $K$ такого, что $f_{K}<\omega$, любого натурального $n>0$ существует и единственное неразветвленное расиирение $L \geqslant K$ maкое, что $f_{L / K}=n$.

ДокАЗАТЕЛЬство. Пусть $L-$ поле такое, что $G_{L}=d_{K}^{-1}(n \widehat{\mathbb{Z}}) ;$ нетрудно проверить, что $L$ искомое.

ЗАмечАниЕ 4 . Если $K \leqslant L \leqslant M, f_{M}<\omega$ (следовательно, и $\left.f_{K}, f_{L / K}<\omega\right)$, $L$ и $M$ - расширения Галуа поля $K$, то $M$ является неразветвленным расширением $L$ тогда и только тогда, когда группа $G(M / K)$ естественно изоморфна расслоенному произведению $G(L / K) \times_{\mathbb{Z}_{L / K}} \mathbb{Z}_{M / K}($ см. диаграмму $(*))$. Этот изоморфизм определен следуюшим образом: элементу $\sigma \in G(M / K)$ ставится в соответствие элемент $\left\langle\pi_{L}^{M}(\sigma), d_{K}^{M}(\sigma)\right\rangle=\left\langle\sigma \uparrow L, d_{K}^{M}(\sigma)\right\rangle \in G(L / K) \times_{\mathbb{Z}_{L / K}} \mathbb{Z}_{M / K}$ (cм. [5; 2.1 , лемма 4] и замечание 3$)$.

Далее будем предполагать, что все рассматриваемые (промежуточные) поля являются конечными расширениями поля $k$.

Зафиксируем образуюшую $\Phi$ групшы $\widehat{\mathbb{Z}}(\widehat{\mathbb{Z}}=\overline{\langle\Phi\rangle})$.

Пусть $K \leqslant L$ - расширение Галуа (по нашему соглашению $L$ (и $K$ ) - конечное расширение $k$ ); пусть $L_{u}^{K}$ - наибольшее неразветвленное подрасширение $L$; тогда 
$G\left(L_{u}^{K} / K\right) \simeq \mathbb{Z}_{L / K}, G\left(L / L_{u}^{K}\right)=I_{K}^{L}$. Обозначим через $\Phi_{K}^{L}$ образ элемента $\Phi$ в групше $\mathbb{Z}_{L / K}=\widehat{\mathbb{Z}} / d_{K}\left(G_{L}\right) ; \Phi_{K}^{L}$ является образующим циклической группы $\mathbb{Z}_{L / K}$. Тогда для любого элемента $\sigma \in G(L / K)$ найдется единственное $m, 0<m \leqslant$ $f_{L / K}=\left|\mathbb{Z}_{L / K}\right|$, такое, что $\sigma \uparrow L_{u}^{K}=\left(\Phi_{K}^{L}\right)^{m} ;$ обозначим это $m$ через $\partial_{K}^{L} \sigma$ (иногда просто $\partial \sigma)$.

Определим теперь множество $\operatorname{Frob}(L / K) \subseteq G(L / K)$ әлементов Фробениуса группы $G(L / K)$ : для $\sigma \in G(L / K) \sigma \in \operatorname{Frob}(L / K)$ тогда и только тогда, когда вьполнены следующие условия:

1) $\langle\sigma\rangle \cap I_{K}^{L}=\langle 1\rangle$;

2) $|\sigma| \times \partial \sigma=f_{L / K}$.

Из условия 1) вытекает, что $|\sigma|=|\sigma| L_{u}^{K} \mid$ и что $L$ является неразветвленным расширением поля $L^{\sigma}$.

Отметим ряд просто проверяемых свойств фробениусовых элементов.

1. Для $\sigma \in G(L / K) \sigma \in \operatorname{Frob}(L / K) \Longleftrightarrow\langle\sigma\rangle \cap I_{K}^{L}=\langle 1\rangle$ и $\partial_{K}^{L} \sigma$ делит $f_{L / K}$.

2. Если $\sigma \in \operatorname{Frob}(L / K), \tau \in G(L / K)$, то $\sigma^{\tau}\left(=\tau^{-1} \sigma \tau\right) \in \operatorname{Frob}(L / K)$ (и $\left.\partial_{K}^{L}\left(\sigma^{\tau}\right)=\partial_{K}^{L} \sigma\right)$.

3. Если $\sigma \in \operatorname{Frob}(L / K), m$ делит $|\sigma|$, то $\sigma^{m} \in \operatorname{Frob}(L / K)$ и $\partial_{K}^{L} \sigma^{m}=m \partial_{K}^{L} \sigma$.

4. Если $K \leqslant K^{\prime} \leqslant L_{u}^{K}$, то $\operatorname{Frob}\left(L / K^{\prime}\right)=\operatorname{Frob}(L / K) \cap G\left(L / K^{\prime}\right)\left(G\left(L / K^{\prime}\right) \leqslant\right.$ $G(L / K))$.

5. Если $\psi \in \operatorname{Frob}(L / K), K^{\prime} \rightleftharpoons\left(L_{u}^{K}\right)^{\psi}$, то $\psi \in \operatorname{Frob}\left(L / K^{\prime}\right)$ и $\partial_{K^{\prime}}^{L} \psi=1$.

Пусть $K \leqslant L \leqslant M, L$ и $M$ - расширения Галуа поля $K$ и $M$ - неразветвленное расширение $L$.

Элемент $\sigma_{0} \in \operatorname{Frob}(M / K)$ назовем подвемом элемента $\sigma \in G(L / K)$, если $\sigma_{0} \uparrow L=\sigma$ и $\partial_{K}^{M} \sigma_{0}=\partial_{K}^{L} \sigma$.

Лемма 1. Для әлемента $\sigma \in G(L / K)$ существует не более одного подвема в $\operatorname{Frob}(M / K)$.

ДоказАТЕльство. Так как $M$-неразветвленное расширение $L$, то $G(M / K) \simeq$ $G(L / K) \times_{\mathbb{Z}_{L / K}} \mathbb{Z}_{M / K}$. Отсюда следует, что отображение ограничения $\sigma \longmapsto \sigma \uparrow L$ индуцирует изоморфизм групп $I_{K}^{M}$ и $I_{K}^{L}$.

Пусть $\sigma_{0}, \sigma_{1} \in \operatorname{Frob}(M / K)$ являются подъемами элемента $\sigma \in G(L / K)$. Тогда $\partial_{K}^{M} \sigma_{0}=\partial_{K}^{M} \sigma_{1}\left(=\partial_{K}^{L} \sigma\right)$ и, следовательно, $\sigma_{0}=\sigma_{1} \tau$ для $\tau\left(\rightleftharpoons \sigma_{1}^{-1} \sigma_{0}\right) \in I_{K}^{M}$. Так как $\sigma_{0} \uparrow L=\sigma=\sigma_{1} \uparrow L$, то $\sigma_{0} \uparrow L=\left(\sigma_{1} \uparrow L\right)\left(\tau \uparrow L_{1}\right)$ влечет, что $\tau \uparrow L=1$; отсюда $\tau=1$ и $\sigma_{0}=\sigma_{1}$.

ЗАмЕчАнИЕ 5. Проведенное рассуждение устанавливает более общий результат: если $\sigma_{0}, \sigma_{1} \in \operatorname{Frob}(M / K), \partial_{K}^{M} \sigma_{0}=\partial_{K}^{M} \sigma_{1} u \sigma_{0} \uparrow L=\sigma_{1} \uparrow L, m o \sigma_{0}=\sigma_{1}$.

Пусть $K \leqslant L \leqslant M \leqslant M^{\prime}$ - башня полей такая, что $L, M$ и $M^{\prime}$ являются расширениями Галуа поля $K ; M$ и $M^{\prime}$ являются неразветвленными расширениями $L$ (тогда $M^{\prime}$ является неразветвленным расширением $M$ ).

Лемма 2. Если $\sigma \in G(L / K) ; \sigma_{0} \in \operatorname{Frob}(M / K)$ u $\sigma_{0}^{\prime} \in \operatorname{Frob}\left(M^{\prime} / K\right)$ являются подбемами элемента $\sigma$, то $\sigma_{0}^{\prime} \uparrow M=\sigma_{0}$.

ДокАЗАТЕЛЬство. Так как $M^{\prime}$ и $M$ являются неразветвленными расширениями $L$, то имеют место изоморфизмы (представления) $G\left(M^{\prime} / K\right) \simeq$ $G(L / K) \times_{\mathbb{Z}_{L / K}} \mathbb{Z}_{M^{\prime} / K}$ и $G(M / K) \simeq G(L / K) \times_{\mathbb{Z}_{L / K}} \mathbb{Z}_{M / K}$, а эпиморфизм 
$\pi_{M}^{M^{\prime}}=\uparrow M: G\left(M^{\prime} / K\right) \longrightarrow G(M / K)$ реализуется на этих представлениях так: $\pi_{M}^{M^{\prime}}\left(\left\langle\sigma, \xi^{\prime}\right\rangle\right)=\left\langle\sigma, \bar{\pi}_{M}^{M^{\prime}}\left(\xi^{\prime}\right)\right\rangle$ для $\sigma \in G(L / K), \xi^{\prime} \in \mathbb{Z}_{M^{\prime} / K}$. Заметим, что $\mathbb{Z}_{M^{\prime} / K}=\left\langle\Phi_{K}^{M^{\prime}}\right\rangle, \mathbb{Z}_{M / K}=\left\langle\Phi_{K}^{M}\right\rangle$, а эпиморфизм $\bar{\pi}_{M}^{M^{\prime}}: \mathbb{Z}_{M^{\prime} / K} \longrightarrow \mathbb{Z}_{M / K}$ определен на образуюшей $\Phi_{K}^{M^{\prime}}$ так: $\bar{\pi}_{K}^{M^{\prime}} \Phi_{K}^{M^{\prime}}=\Phi_{K}^{M}$.

Воспользуемся этим для доказательства леммы. Элементу $\sigma_{0}^{\prime}$ в представлении $G\left(M^{\prime} / K\right) \simeq G(L / K) \times_{\mathbb{Z}_{L / K}} \mathbb{Z}_{M^{\prime} / K}$ соответствует элемент $\left\langle\sigma_{0}^{\prime} \uparrow L, d_{K}^{M^{\prime}}\left(\sigma_{0}^{\prime}\right)\right\rangle$; но $\sigma_{0}^{\prime} \uparrow L=\sigma$, а $d_{K}^{M^{\prime}}\left(\sigma_{0}^{\prime}\right)=\left(\Phi_{K}^{M^{\prime}}\right)^{\partial_{K}^{M^{\prime}}}\left(\sigma_{0}^{\prime}\right)=\left(\Phi_{K}^{M^{\prime}}\right) \partial_{K}^{L}(\sigma) ;$ аналогично, элементу $\sigma_{0}$ в представлении $G(M / K) \simeq G(L / K) \times_{\mathbb{Z}_{L / K}} \mathbb{Z}_{M / K}$ соответствует элемент $\left\langle\sigma,\left(\Phi_{K}^{M}\right)^{\partial_{K}^{L}(\sigma)}\right\rangle$; элементу $\pi_{M}^{M^{\prime}}\left(\sigma_{0}\right)=\sigma_{0} \uparrow M$ соответствует элемент $\left\langle\sigma, \bar{\pi}_{M}^{M^{\prime}}\left(\Phi_{K}^{M^{\prime}}\right)^{\partial_{K}^{L}(\sigma)}\right\rangle=\left\langle\sigma,\left(\Phi_{K}^{M}\right)^{\partial_{K}^{L}(\sigma)}\right\rangle$. Отсюда и получается, что $\sigma_{0}^{\prime}\left\lceil M=\sigma_{0}\right.$.

Элементы $\sigma_{0}, \sigma_{1} \in \operatorname{Frob}(L / K)$ назовем когерентныцми, если $\sigma_{0} \sigma_{1} \in \operatorname{Frob}(L / K)$ и $\partial_{K}^{L}\left(\sigma_{0} \sigma_{1}\right)=\partial_{K}^{L}\left(\sigma_{0}\right)+\partial_{K}^{L}\left(\sigma_{1}\right)$.

ПРЕДЛОЖЕНИЕ 3 . Пусть $L \geqslant K$ - расиирение Галуа, тогда существует натуральное число $n_{L / K}>0$ такое, что для любого неразветвленного расширения $M \geqslant L$ такого, что $n_{L / K}$ делит $[M: L]$, справедливы следующие свойства:

1) существует фробениусов әлемент $\psi \in \operatorname{Frob}(M / K)$ такой, что $\partial_{K}^{M} \psi=1$;

2) для любого автоморфизма $\sigma \in G(L / K)$ существует его подвем $\sigma_{0} \in$ $\operatorname{Frob}(M / K)$

3) если $\sigma_{0}, \sigma_{1} \in \operatorname{Frob}(M / K)$ - подгемы әлементов из $G(L / K)$, то они когерентны.

Лемма 3. Пусть $M$ - такое неразветвленное расширение $L$, что период $\omega(G(L / K))$ группы $G(L / K)$ делит степень $m=[M: L]$ расширения $M \geqslant L$. Тогда в $\operatorname{Frob}(M / K)$ существует әлемент $\psi$ такой, что $\partial_{K}^{M} \psi=1$.

ДоКАЗАТЕЛЬСТво. Имеет место изоморфизм $G(M / K) \simeq G(L / K) \times_{\mathbb{Z}_{L / K}} \mathbb{Z}_{M / K}$. Так как $d_{K}^{L}: G(L / K) \longrightarrow \mathbb{Z}_{L / K}-$ эпиморфизм, то в $G(L / K)$ сушествует $\sigma_{0}$ такой, что $d_{K}^{L}\left(\sigma_{0}\right)=\Phi_{K}^{L}$. Заметим, что тогда элемент $\left\langle\sigma_{0}, \Phi_{K}^{M}\right\rangle \in G(L / K) \times_{\mathbb{Z}_{L / K}} \mathbb{Z}_{M / K}$ $\left(\mathbb{Z}_{M / K}=\left\langle\Phi_{K}^{M}\right\rangle, \mathbb{Z}_{L / K}=\left\langle\Phi_{K}^{L}\right\rangle\right)$. Проверим, что этот элемент имеет порядок $f_{M / K}$. Действительно,

$$
\left\langle\sigma_{0}, \Phi_{K}^{M}\right\rangle^{f_{M / K}}=\left\langle\sigma_{0}^{f_{M / K}},\left(\Phi_{K}^{M}\right)^{f_{M / K}}\right\rangle=\langle 1,1\rangle,
$$

так как $\left|\sigma_{0}\right|$ делит $\omega(G(L / K)), \omega(G(L / K))$ делит $m, m$ делит $f_{M / K}\left(m=f_{M / K}\right)$ и, следовательно, $\sigma_{0}^{f_{M / K}}=1 ;\left(\Phi_{K}^{M}\right)^{f_{M / K}}=1$, так как $\left|\mathbb{Z}_{M / K}\right|=f_{M / K}$. Если $\psi \in G(M / K)$ соответствует элементу $\left\langle\sigma_{0}, \Phi_{K}^{M}\right\rangle$, то $\psi$ искомый.

Установим теперь одну теоретико-групповую лемму.

Пусть $G$ - конечная группа, $H \unlhd G$ - нормальная подгруппа; $\omega(H)$ - период группы $H ; \omega($ Aut $H)$ - период группы всех автоморфизмов группы $H ; \Omega(H) \rightleftharpoons$ $\omega(H) \cdot \omega($ Aut $H)$.

ЛЕмма 4. Если $g \in G,\langle g\rangle \cap H=\langle 1\rangle$, и порядок $m=|g|$ әлемента $g$ делится на $\Omega(H)$, то для любого $h \in H$ порядок әлемента $g h$ равен $m(u\langle g h\rangle \cap$ $H=\langle 1\rangle)$. 
ДокАЗАТЕльство. Из рассмотрения образов элементов $g$ и $g h$ в факторгруппе $G / H$ легко следует, что $|g|=|g H|=|g h H| \leqslant|g h|$. Поэтому достаточно установить, что $(g h)^{m}=1$. Имеем $(g h)^{m}=g^{m} \cdot h^{g^{m-1}+g^{m-2}+\cdots+g+1}$.

Пусть $n=\omega($ Aut $H)$ и $m=n \cdot k$; тогда $\omega(H)$ делит $k$. Представим выражение $g_{m}=g^{m-1}+g^{m-2}+\cdots+g+1$ так:

$$
\begin{aligned}
g_{m}= & \left(g^{n(k-1)+(n-1)}+g^{n(k-1)+(n-2)}+\cdots+g^{n(k-1)+1}+g^{n(k-1)+0}\right) \\
& +\left(g^{n(k-2)+(n-1)}+g^{n(k-2)+(n-2)}+\cdots+g^{n(k-2)+1}+g^{n(k-2)+0}\right) \\
& +\cdots+\left(g^{n \cdot 0+(n-1)}+g^{n \cdot 0+(n-2)}+\cdots+g^{n \cdot 0+1}+g^{n \cdot 0+0}\right) .
\end{aligned}
$$

Далее заметим, что $h^{g^{n \cdot i+j}}=h^{g^{j}}$; действительно, $h^{g^{n \cdot i+j}}=\left(h^{g^{n \cdot i}}\right)^{g^{j}} ; h^{g^{n \cdot i}}=$ $h^{g^{i \cdot n}}=h^{\left(g^{i}\right)^{n}}=h$, так как $n=\omega($ Aut $H)$ и образ элемента $g^{i}$ в группе Aut $H$ имеет порядок, делящийся на $n$. Итак, $h^{g^{n \cdot i+j}}=\left(h^{g^{n \cdot i}}\right)^{g^{j}}=h^{g^{j}}, \mathrm{a}$

$$
\begin{aligned}
h^{g_{m}}= & h^{g^{n(k-1)+(n-1)}+\cdots+g^{n(k-1)+0}} \cdot h^{g^{n(k-2)+(n-1)}+\cdots+g^{n(k-2)+0}} \\
& \cdots h^{g^{n-1}+g^{n-2}+\cdots+1}=\left(h^{g_{n}}\right)^{k}
\end{aligned}
$$

но $h^{g_{n}} \in H$, а $\omega(H)$ делит $k$; следовательно, $\left(h^{g_{n}}\right)^{k}=1$ и $h^{g_{m}}=1$. Вместе с соотношением $g^{m}=1$ имеем $(g h)^{m}=g^{m} h^{g_{m}}=1$.

СлЕДСТВИЕ 1. Пусть $L \geqslant K$ - расширение Галуа, $\sigma \in \operatorname{Frob}(L / K) ;$ если $\Omega\left(I_{K}^{L}\right)$ делит $|\sigma|$, то $\sigma \tau \in \operatorname{Frob}(L / K)$ для любого әлемента $\tau \in I_{K}^{L}$.

ДоКАЗАТЕЛЬСТво ПРЕДЛОЖЕНИЯ 3. Для любого натурального $m>0$ через $\langle m\rangle$ обозначим наименьшее обшее кратное чисел $1,2, \ldots, m$ :

$$
\langle m\rangle=\prod_{p \leqslant m, p \text { простое }} p^{\left[\log _{p} m\right]} .
$$

Полагаем $n_{L / K} \rightleftharpoons\left\langle 2 \cdot f_{L / K}\right\rangle \cdot \omega(G(L / K)) \cdot \Omega\left(I_{K}^{L}\right)$ и проверим, что это число удовлетворяет предложению 3.

Пусть $M$ - неразветвленное расширение $L$ такое, что $n_{L / K}$ делит $[M: L]$. Так как $\omega(G(L / K))$ делит $n_{L / K}$, то по лемме 3 в $\operatorname{Frob}(M / K)$ найдется элемент $\sigma_{0}$ такой, что $\partial_{K}^{M}\left(\sigma_{0}\right)=1$; заметим, что порядок $\left|\sigma_{0}\right|=f_{M / L}=f_{M / K} \cdot f_{L / K}$ элемента $\sigma_{0}$ делится на $n_{L / K}$.

Пусть $\sigma \in G(L / K), m \rightleftharpoons \partial_{K}^{L}(\sigma)$; тогда $d_{K}^{L}(\sigma)=d_{K}^{L}\left(\left(\sigma_{0} \uparrow L\right)^{m}\right)$; следовательно, $\sigma=\left(\sigma_{0} \uparrow L\right)^{m} \tau$ для подходящего элемента $\tau \in I_{K}^{L}=\operatorname{Ker} d_{K}^{L}$. Пусть $\tau_{0} \in I_{K}^{M}$ такой, что $\tau_{0} \uparrow L=\tau$. Тогда имеет место равенство $\left(\sigma_{0}^{m} \tau_{0}\right) \uparrow L=\sigma$. Проверим, что $\sigma_{0}^{m} \tau_{0} \in \operatorname{Frob}(M / K)$. Так как $m$ делит порядок $\sigma$ и, следовательно, $\omega(G(L / K))$, то по свойству 3 элементов Фробениуса $\sigma_{0}^{m} \in \operatorname{Frob}(M / K)$, $\left|\sigma_{0}^{m}\right|=|\sigma| \cdot m^{-1}$. Но тогда $\Omega\left(I_{K}^{L}\right)$ делит $\left|\sigma_{0}^{m}\right| ;$ группы $I_{K}^{L}$ и $I_{K}^{M}$ изоморфны $(M-$ неразветвленное расширение $L)$; тогда по следствию $1 \sigma_{0}^{m} \tau_{0} \in \operatorname{Frob}(M / K)$; кроме того, $\partial_{K}^{M}\left(\sigma_{0}^{m} \tau_{0}\right)=m=\partial_{K}^{L}(\sigma)$ и $\sigma_{0}^{m} \tau_{0}$ является подъемом элемента $\sigma$. Свойство 2$)$ предложения установлено.

Для доказательства свойства 3) установим более общее утверждение: если $\sigma_{1}, \sigma_{2} \in \operatorname{Frob}(M / K)$ и $m_{i} \rightleftharpoons \partial_{K}^{M} \sigma_{i} \leqslant f_{L / K}, i=1,2$, то $\sigma_{1}, \sigma_{2}$ когерентны. Для подходящих элементов $\tau_{1}, \tau_{2} \in I_{K}^{M}$ имеют место равенства $\sigma_{1}=\sigma_{0}^{m_{1}} \tau_{1}$, 
$\sigma_{2}=\sigma_{0}^{m_{1}} \tau_{2}$; тогда $\sigma_{1} \sigma_{2}=\sigma_{0}^{m_{1}+m_{2}} \tau_{3}$, где $\tau_{3}=\tau_{1}^{\sigma_{0}^{m_{2}}} \tau_{2} \in I_{K}^{M}$. Так как $m_{1}+m_{2} \leqslant$ $2 f_{L / K}$, то $\left(m_{1}+m_{2}\right) \Omega\left(I_{K}^{L}\right)$ делит $n_{L / K}$, следовательно, $\sigma_{0}^{m_{1}+m_{2}} \in \operatorname{Frob}(M / K)$ и $\Omega\left(I_{K}^{M}\right)=\Omega\left(I_{K}^{L}\right)$ делит $\left|\sigma_{0}^{m_{1}+m_{2}}\right| ;$ тогда по следствию $1 \sigma_{0}^{m_{1}+m_{2}} \tau_{3} \in \operatorname{Frob}(M / K)$ и $\partial_{K}^{M}\left(\sigma_{0}^{m_{1}+m_{2}} \tau_{3}\right)=\partial_{K}^{M}\left(\sigma_{0}^{m_{1}+m_{2}}\right)=m_{1}+m_{2}$. Следовательно, элементы $\sigma_{1}$ и $\sigma_{2}$ когерентны.

Предложение 3 доказано.

ЗАмечание 6 . Утверждение 1) предложения 3 влечет, что эпиморфизм $d_{K}^{M}: G(M / K) \longrightarrow \mathbb{Z}_{M / K}$ расшепляется.

Пусть теперь задан непрерывный мультипликативный $G$-модуль $A$, т.е. мультипликативная абелева группа $A$, на которой элементы $\sigma \in G$ действуют как автоморфизмы справа: $a \longmapsto a^{\sigma}, a \in A$, так, что выполнены соотношения:

i) $a^{1}=a$,

ii) $(a b)^{\sigma}=a^{\sigma} b^{\sigma}$,

iii) $a^{\sigma \tau}=\left(a^{\sigma}\right)^{\tau}$

iv) $A=\bigcup_{[K: k]<\omega} A_{K}$, где $A_{K} \rightleftharpoons\left\{a \mid a \in A, a^{\sigma}=a\right.$ для всех $\left.\sigma \in G_{K}\right\}$.

Для каждого расширения $L \geqslant K$ имеем $A_{K} \leqslant A_{L}$, и если $[L: K]<\omega$, то определено отображение нормы $N_{L / K}: A_{L} \longrightarrow A_{K}$ :

$$
N_{L / K}(a) \rightleftharpoons \prod_{\sigma} a^{\sigma}
$$

где $\sigma$ пробегает систему представителей $G_{L} \backslash G_{K}\left(G_{K}=\dot{\bigcup} G_{L} \sigma\right)$. Если $L \geqslant K-$ расширение Галуа, то $A_{L}$ является $G(L / K)$-модулем и $A_{L}^{G(L / K)}=A_{K}$.

Замечание 7 . Если $L \geqslant K$ - циклическое расширение, $G(L / K)=\langle\sigma\rangle$ и $n \rightleftharpoons$ $[L / K]$, то для $a \in A_{L} \quad N_{L / K}(a)=a^{\sigma_{n}}=a^{\sigma^{n-1}+\sigma^{n-2}+\cdots+\sigma+1}$.

В теории полей классов важны следующие группы (связанные с $G$ и модулем $A$ ):

$$
\begin{aligned}
H^{0}\left(G(L / K), A_{L}\right) & \rightleftharpoons A_{K} / N_{L / K}\left(A_{L}\right), \\
H^{-1}\left(G(L / K), A_{L}\right) & { }_{N_{L / K}} A_{L} / I_{G(L / K)} A_{L},
\end{aligned}
$$

где $N_{L / K} A_{L} \rightleftharpoons\left\{a \mid a \in A_{L}, N_{L / K}(a)=1\right\}$, а $I_{G(L / K)} A_{L}$ - подгруппа группы $N_{L / K} A_{L}$, порожденная всеми элементами вида $a^{\sigma-1}=a^{\sigma} a^{-1}$, где $a \in A_{L}, \sigma \in$ $G(L / K)$. Если $G(L / K)$ циклическая, а $\sigma$ является образующей, то

$$
I_{G(L / K)} A_{L}=A_{L}^{\sigma-1} \rightleftharpoons\left\{a^{\sigma-1} \mid a \in A_{L}\right\},
$$

а именно формальное равенство

$$
\sigma^{k}-1=\left(\sigma^{k-1}+\cdots+\sigma+1\right)(\sigma-1)=\sigma_{k}(\sigma-1)
$$

влечет, что $a^{\sigma^{k}-1}=b^{\sigma-1}$, где $b \rightleftharpoons a^{\sigma_{k}}$.

Гензелевым нормированиемгруппы $A_{k}$ по отношению к эпиморфизму $d: G \longrightarrow$ $\widehat{\mathbb{Z}}$ называется гомоморфизм

$$
v: A_{k} \longrightarrow \widehat{\mathbb{Z}}
$$

удовлетворяющий следующим свойствам:

(i) $\Phi \in Z \rightleftharpoons v\left(A_{k}\right)$ и $Z / n Z \simeq \widehat{\mathbb{Z}} / n \widehat{\mathbb{Z}}$ для всех $n>0$;

(ii) $v\left(N_{K / k} A_{k}\right)=f_{K} Z$ для всех (конечных) расширений $K \geqslant k$. 
ПРЕДЛОЖЕНИЕ $4[2 ;$ предложение (4.7)]. Для каждого конечного расширения $K \geqslant k$ формула

$$
v_{K} \rightleftharpoons f_{K}^{-1} v \cdot N_{K / k}: A_{K} \longrightarrow Z
$$

определяет эпиморфизм, удовлетворяющий следующим свойствам:

(i) $v_{K}=v_{\sigma(K)} \cdot \sigma$ для всех $\sigma \in G$;

(ii) для всякого конечного расширения $L \geqslant K$ диаграмма

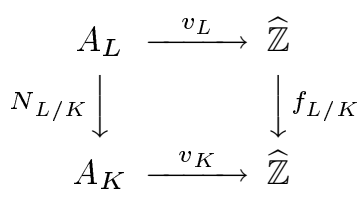

коммутативна.

Простым әлементом групшы $A_{K}$ называется любой $\pi_{K} \in A_{K}$ такой, что $v_{K}\left(\pi_{K}\right)=\Phi$. Полагаем $U_{K} \rightleftharpoons\left\{u \mid u \in A_{K}, v_{K}(u)=0\right\}$.

Для неразветвленного расширения $L \geqslant K$ из предложения 4 следует, что $v_{L} \uparrow A_{K}=v_{K}$; в частности, простой элемент $A_{K}$ будет и простым элементом $A_{L}$. Если $L \geqslant K$ вполне разветвлено и $\pi_{L}-$ простой элемент $A_{L}$, то $\pi_{K} \rightleftharpoons N_{L / K}\left(\pi_{L}\right)$ - простой элемент $A_{K}$.

Предположим, что выполнена следующая аксиома $U[2$; аксиома (5.1)]:

$$
H^{i}\left(G(L / K), U_{L}\right)=1, \quad i=0,-1
$$

для любого неразветвленного (конечного) расширения Галуа $L \geqslant K$.

Определим отображсение Нейкирха $\nu_{L / K}: \operatorname{Frob}(L / K) \longrightarrow A_{K} / N_{L / K}\left(U_{L}\right)$ для конечного расширения Галуа $L \geqslant K$ следуюшим образом: для $\sigma \in \operatorname{Frob}(L / K)$ пусть $\Sigma \rightleftharpoons L^{\sigma}, \pi_{\Sigma}-$ простой элемент $A_{\Sigma}$, тогда

$$
\nu_{L / K}(\sigma) \rightleftharpoons N_{\Sigma / K}\left(\pi_{\Sigma}\right) \cdot N_{L / K}\left(U_{L}\right)
$$

Проверим корректность определения. Пусть $\pi_{\Sigma}^{\prime}-$ другой простой элемент $A_{\Sigma}$; тогда $\pi_{\Sigma}^{\prime}=\pi_{\Sigma} u$ для $u \in U_{\Sigma}$. Так как $L$ - неразветвленное расширение $\Sigma=L^{\sigma}$, то по аксиоме $U$ найдется $v \in U_{L}$ такой, что $N_{L / \Sigma}(v)=u$. Тогда имеем

$$
\begin{aligned}
N_{\Sigma / K}\left(\pi_{\Sigma}^{\prime}\right) & =N_{\Sigma / K}\left(\pi_{\Sigma} u\right)=N_{\Sigma / K}\left(\pi_{\Sigma}\right) \cdot N_{\Sigma / K}(u) \\
& =N_{\Sigma / K}\left(\pi_{\Sigma}\right) N_{\Sigma / K} N_{L / \Sigma}(v)=N_{\Sigma / K}\left(\pi_{\Sigma}\right) N_{L / K}(v)
\end{aligned}
$$

и, следовательно,

$$
N_{\Sigma / K}\left(\pi_{\Sigma}^{\prime}\right) \cdot N_{L / K}\left(U_{K}\right)=N_{\Sigma / K}\left(\pi_{\Sigma}\right) \cdot N_{L / K}\left(U_{K}\right)
$$

Корректность определения отображения $\nu_{L / K}$ проверена.

ПРЕДЛОЖЕНИЕ 5. Пусть $K \leqslant L \leqslant M, L$ и $M$ - расширения Галуа поля $K ;$ пусть $\psi \in \operatorname{Frob}(M / K) ;$ если $\psi \rightleftharpoons \psi_{0} \uparrow L \in \operatorname{Frob}(L / K), \operatorname{mo} \nu_{L / K}(\psi)=$ $\nu_{M / K}\left(\psi_{0}\right) N_{L / K}\left(U_{L}\right)$. 


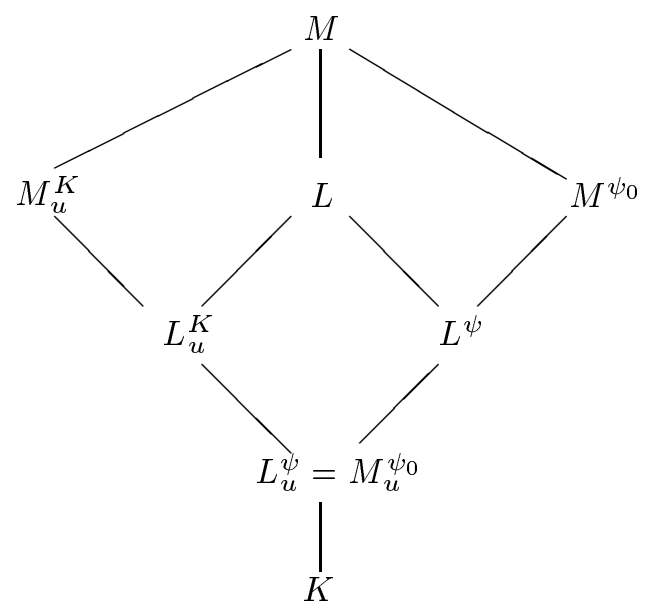

Рис. 2

ДокаЗАТЕЛЬСтво. Заметим, что $M^{\psi_{0}}$ - вполне разветвленное расширение $L^{\psi}$. Если $\pi_{0}$ - простой элемент $A_{M^{\psi_{0}}}$, то $\pi \rightleftharpoons N_{M^{\psi_{0} / L}}\left(\pi_{0}\right)$ - простой элемент $A_{L \psi}$. Тогда имеем

$$
\begin{aligned}
\nu_{L / K}(\psi) & =N_{L \psi / K}(\pi) \cdot N_{L / K}\left(U_{L}\right)=N_{L \psi / K}\left(N_{M^{\psi_{0} / L \psi}}\left(\pi_{0}\right)\right) \cdot N_{L / K}\left(U_{L}\right) \\
& =N_{M^{\psi_{0} / K}}\left(\pi_{0}\right) \cdot N_{L / K}\left(U_{L}\right)=\nu_{M / K}\left(\psi_{0}\right) \cdot N_{L / K}\left(U_{L}\right) .
\end{aligned}
$$

Заметим, что $N_{M / K}\left(U_{M}\right) \leqslant N_{L / K}\left(U_{L}\right)$.

ЗАмечание 8. Если $M$ - неразветвленное расширение $L$, то $N_{M / K}\left(U_{M}\right)=$ $N_{L / K} N_{M / L}\left(U_{M}\right)=N_{L / K}\left(U_{L}\right)$ и тогда (в условиях предложения 5$) \nu_{L / K}(\psi)=$ $\nu_{M / L}\left(\psi_{0}\right)$.

ЗАмЕчАниЕ 9. Если $M$ - вполне разветвленное расширение, то условие предложения 5 всегда выполнено:

$$
\psi_{0} \in \operatorname{Frob}(M / K) \Longrightarrow \psi_{0}\lceil L \in \operatorname{Frob}(L / K) .
$$

В предположении, что в $\operatorname{Frob}(L / K)$ сушествует элемент $\psi$ такой, что $\partial_{K}^{L} \psi=1$, установим ряд важных свойств отображения $\nu_{L} / K$.

Пусть $\psi^{\prime} \in \operatorname{Frob}(L / K), m \rightleftharpoons \partial_{K}^{L} \psi^{\prime}$; тогда $\psi^{\prime}=\psi^{m} \tau$ для подходящего $\tau(\rightleftharpoons$ $\left.\psi^{-m} \psi^{\prime}\right) \in H=I_{K}^{L}$. Пусть $K^{\prime} \rightleftharpoons L_{u}^{K \psi^{\prime}}$; тогда $\psi^{\prime} \in \operatorname{Frob}\left(L / K^{\prime}\right)$ и $\partial_{K^{\prime}}^{L} \psi^{\prime}=1$; $G\left(K^{\prime} / K\right)=\left\langle\psi \mid K^{\prime}\right\rangle ;\left[K^{\prime}: K\right]=m$ и $N_{K^{\prime} / K}(a)=a^{\psi_{m}}$ для $a \in A_{K^{\prime}}$.

Так как $N_{L / K}\left(U_{L}\right)=N_{K^{\prime} / K} N_{L / K^{\prime}}\left(U_{L}\right)$, то $N_{K^{\prime} / K}$ индуцирует гомоморфизм $\widetilde{N}_{K^{\prime} / K}: A_{K^{\prime}} / N_{L / K^{\prime}}\left(U_{L}\right) \longrightarrow A_{K} / N_{L / K}\left(U_{L}\right)$.

Лемма 5. Имеет место следующее соотношение:

$$
\widetilde{N}_{K^{\prime} / K} \nu_{L / K^{\prime}}\left(\psi^{\prime}\right)=\nu_{L / K}\left(\psi^{\prime}\right) .
$$

ДокАЗАТЕльСтво. Пусть $\pi$ - простой элемент $A_{\Sigma}$, где $\Sigma=L^{\psi^{\prime}}$; тогда равенства $N_{L / K}(\pi)=N_{K^{\prime} / K} N_{L / K}(\pi), \nu_{L / K}\left(\psi^{\prime}\right)=N_{L / K}(\pi) N_{L / K}\left(U_{L}\right), \nu_{L / K^{\prime}}=$ $N_{L / K^{\prime}}(\pi) N_{L / K^{\prime}}\left(U_{L}\right)$ и влекут нужное соотношение. 


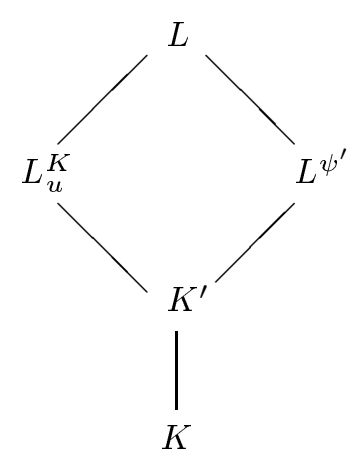

Рис. 3

ЛЕмма 6. Если $m$ делит $|\psi|$ (m.e. $\left.\psi^{m} \in \operatorname{Frob}(L / K)\right)$, mo $\nu_{L / K}\left(\psi^{m}\right)=$ $\nu_{L / K}(\psi)^{m}$.

ДокаЗАТЕЛЬство. Пусть $\pi_{\psi}$ - простой элемент $A_{L \psi}$; тогда $\pi_{\psi}$ является и простым элементом $A_{L}^{\psi^{m}}\left(L^{\psi^{m}}\right.$ - неразветвленное расширение $\left.L^{\psi},\left[L^{\psi^{m}}: L^{\psi}\right]=m\right)$ и $N_{L^{m} / K}\left(\pi_{\psi}\right)=N_{L \psi / K} N_{L_{\psi} m / L_{\psi}}\left(\pi_{\psi}\right)=N_{L^{\psi} / K}\left(\pi_{\psi}^{m}\right)=N_{L^{\psi} / K}\left(\pi_{\psi}\right)^{m}$. Отсюда и следует заключение леммы.

Пусть $L_{u}^{K}$ - наибольшее неразветвленное расширение $K$ в $L$; заметим, что $L_{u}^{K} \|_{K} L^{\psi}$ и $L=L_{u}^{K} \otimes_{K} L^{\psi}$; полагаем $H \rightleftharpoons G\left(L / L_{u}^{K}\right)\left(H=I_{K}^{L}\right) ;$ тогда $G(L / K)=\langle\psi\rangle<H$.

Введем в рассмотрение группу $H_{K}^{-1}\left(H, U_{L}\right)$, определенную так: пусть $V_{K}\left(L / L_{u}^{K}\right)=\left\langle u^{\tau-1}, v^{1-\psi}\right| u \in U_{L}, \tau \in H, v \in_{N_{L / L_{u}^{K}}} U_{L}\left(=\left\{u \mid u \in U_{L}\right.\right.$, $\left.\left.\left.N_{L / L_{u}^{K}}(u)=1\right\}\right)\right\rangle$ и $H_{K}^{-1}\left(H, U_{L}\right) \rightleftharpoons N_{L / L_{u}^{K}} U_{L} / V_{K}\left(L / L_{u}^{K}\right)$. Нетрудно видеть, что $V_{K}\left(L / L_{u}^{K}\right) \geqslant I_{G\left(L / L_{u}^{K}\right)} U_{L}$, так что имеется естественный эпиморфизм $H^{-1}\left(H, U_{L}\right) \longrightarrow H_{K}^{-1}\left(H, U_{L}\right)$.

ЗАмЕчаниЕ 10 . На группе $H^{-1}\left(H, U_{L}\right)$ определено естественное действие автоморфизма $\psi$ (на самом деле, всей групшы $G(L / K)$ ), и группа $H_{K}^{-1}\left(H, U_{L}\right)$ является наибольшей факторгруппой $H^{-1}\left(H, U_{L}\right)$, на которой действие $\psi$ становится тривиальным.

Обозначим через $N$ норменное отображение $N_{L / L_{u}^{K}}$ и положим $U_{K}^{L} \rightleftharpoons\{u \mid$ $u \in U_{K}$, сушествует $v \in U_{L}$ такой, что $\left.N(v)=u\right\}$. Определим отображение $\rho\left(=\rho_{K}^{L}\right): U_{K}^{L} \longrightarrow H_{K}^{-1}\left(H, U_{L}\right)$ так: для $u \in U_{K}^{L}$ пусть $\rho(u)=v^{1-\psi} V_{K}\left(L / L_{u}^{K}\right)$, где $v \in U_{L}$ такой, что $N(v)=u$.

Лемма 7. Отображение $\rho$ определено корректно, является гомоморфизмом и не зависит от въбора $\psi$.

ДокаЗАТЕЛЬСТво. Пусть $v, v^{\prime} \in U_{L}, N(v)=N\left(v^{\prime}\right)=u$; тогда $v^{\prime}=v w, w \in$ $U_{L}$ и $N(w)=1$; отсюда $\left(v^{\prime}\right)^{1-\psi}=(v w)^{1-\psi}=v^{1-\psi} w^{1-\psi} ;$ но $w^{1-\psi} \in V_{K}\left(L / L_{u}^{K}\right)$, так как $w \in N_{L / L_{u}^{K}} U_{L} \leqslant N_{L / K} U_{L}$. Корректность проверена.

Если $u_{0}, u_{1} \in U_{K}^{L} ; v_{0}, v_{1} \in U_{L}$ таковы, что $N\left(v_{i}\right)=u_{i}$, то $N\left(v_{0} v_{1}\right)=u_{0} u_{1}$ и $\left(v_{0} v_{1}\right)^{1-\psi}=v_{0}^{1-\psi} \cdot v_{1}^{1-\psi}$. Отсюда следует, что $\rho$ - гомоморфизм. 
Пусть $\psi^{\prime} \in \operatorname{Frob}(L / K), \partial_{K}^{L} \psi^{\prime}=1$; тогда $\psi^{\prime}=\psi \tau$ для $\tau\left(\rightleftharpoons \psi^{\prime} \psi^{-1}\right) \in H$; если $v \in$ $U_{L}$ и $u=N(v) \in U_{K}$, то $v^{1-\psi^{\prime}}=v^{1-\psi \tau}=v^{1-\psi+\psi-\psi \tau}=v^{1-\psi}\left(v^{-\psi}\right)^{\tau-1} ; v^{-\psi} \in$ $U_{L},\left(v^{-\psi}\right)^{\tau-1} \in V_{K}\left(L / L_{u}^{K}\right) ;$ следовательно, $v^{1-\psi^{\prime}} V_{K}\left(L / L_{u}^{K}\right)=v^{1-\psi} V_{K}\left(L / L_{u}^{K}\right)$.

Лемма доказана.

ПРЕДЛОЖЕНИЕ 6. Ядром гомоморфизма $\rho: U_{K}^{L} \longrightarrow H_{K}^{-1}\left(H, U_{L}\right)$ является $N_{L / K}\left(U_{L}\right)$.

ДокАЗАТЕльство (рис. 4 показывает диаграмму взаимодействий всех объектов из доказательства предложения). Пусть $w \in U_{L}$ и $u \rightleftharpoons N_{L / K}(w)$. Полагаем $v \rightleftharpoons N_{L / L \psi}(w) \in U_{L \psi}$; тогда $u=N_{L / K}(w)=N_{L \psi / K} N_{L / L \psi}(w)=N_{L \psi / K}(v)=$ $N(v)$ и $v^{1-\psi}=1$, так как $v \in L^{\psi}$.

Отсюда $u \in U_{K}^{L}, \rho(u)=1$ и $N_{L / K}\left(U_{K}\right) \leqslant \operatorname{Ker} \rho$.

Наоборот, пусть $u=N(v) \in U_{K}^{L}, v \in U_{L}$ и $v^{1-\psi} \in V_{K}\left(L / L_{u}^{K}\right)$. Тогда сушествуют $v_{i} \in U_{L}, \tau_{i} \in H, i<k, w \in_{N} U_{L}$ такие, что $v^{1-\psi}=w^{1-\psi} \prod_{i<k} v_{i}^{\tau_{i}-1}$. Так как $N(w)=1$, то $N\left(v w^{-1}\right)=N(v)=u$. Поэтому можно считать, что $w=1$.

Итак, $v^{1-\psi}=\prod_{i<k} v_{i}^{\tau_{i}-1}$. Нужно показать, что $u=N(v) \in N_{L / K}\left(U_{L}\right)$.

Пусть $M$ - неразветвленное расширение $L$ такое, что $n \rightleftharpoons[L: K]$ делит $f$ $(\rightleftharpoons[M: L])$ и в $\operatorname{Frob}(M / K)$ сушествует подъем элемента $\psi$, т.е. такой элемент $\psi^{\prime} \in \operatorname{Frob}(M / K)$, что $\partial_{K}^{M} \psi^{\prime}=\partial_{K}^{L} \psi=1$ и $\psi^{\prime} \uparrow=\psi$. Предложение 3 гарантирует сушествование такого расширения. Пусть $M_{u}^{K}$ - наибольшее неразветвленное расширение $K$ в $M$.

Заметим, что $f_{L / K}$ делит $n$. Полагаем $\sigma \rightleftharpoons\left(\psi^{\prime}\right)^{f_{L / K}}$; тогда $\sigma \uparrow L=\left(\psi^{\prime} \uparrow L\right)^{f_{L / K}}$ $=\psi^{f_{L / K}}=\operatorname{id}_{L}$. Следовательно, $L \leqslant M^{\sigma}$; имеем также соотношения $\left[M: M^{\sigma}\right]=$ $|\sigma|=\left|\psi^{\prime}\right| \cdot f_{L / K}^{-1} ;[M: K]=\left|\psi^{\prime}\right| \cdot\left[M: M_{u}^{K}\right]=\left|\psi^{\prime}\right| \cdot\left[L: L_{u}^{K}\right]=\left|\psi^{\prime}\right||H|=[M: L] \cdot$ $[L: K]=[M: L] \cdot f_{L / K}|H|$; отсюда $[M: L]=\left|\psi^{\prime}\right| \cdot f_{L / K}^{-1}=|\sigma|$, что вместе с $L \leqslant M^{\sigma}$ дает $L=M^{\sigma}$.

Так как $M$ - неразветвленное расширение $L$, то аксиома $U$ гарантирует сушествование элементов $w, w_{i} \in U_{M}, i<k$, таких, что $N_{M / L}(w)=v, N_{M / L}\left(w_{i}\right)=v_{i}$, $i<k$. Имеем $N_{M / L}\left(w^{1-\psi^{\prime}}\right)=N_{M / L}(w)^{1-\psi^{\prime}}=v^{1-\psi^{\prime}}=v^{1-\psi} ; N_{M / L}\left(w_{i}^{\tau_{i}-1}\right)=$ $N_{M / L}\left(w_{i}\right)^{\tau_{i}-1}=v_{i}^{\tau_{i}-1}, i<k$ (здесь предполагается отождествление групп $I_{K}^{M}$ и $\left.I_{K}^{L}=H\right)$. Но тогда имеет место соотношение $w^{1-\psi^{\prime}}=x \cdot \prod_{i<k} w_{i}^{\tau_{i}-1}$ для подходящего $x \in U_{M}$ такого, что $N_{M / L}(x)=1$; по аксиоме $U$ имеем $N_{M / L} U_{M}=$ $I_{G(M / L)} U_{M}$, следовательно, сушествует $y \in U_{M}$ такой, что $x=y^{1-\sigma}\left(L=M^{\sigma}\right)$. Тогда имеют место равенства

$$
w^{1-\psi^{\prime}}=y^{1-\sigma} \cdot \prod w_{i}^{\tau_{i}-1}=y^{1-\psi^{\prime f_{L} / K}} \cdot \prod w_{i}^{\tau_{i}-1}=\left(y^{\psi_{f_{L} / K}^{\prime}}\right)^{1-\psi^{\prime}} \cdot \prod w_{i}^{\tau_{i}-1}
$$

Применяя норменное отображение $N^{\prime} \rightleftharpoons N_{N / M_{u}^{K}}$ (расширяюшее отображение $\left.N=N_{L / L_{u}^{K}}\right)$ к этому равенству, получаем

$$
N^{\prime}\left(w^{1-\psi^{\prime}}\right)=N^{\prime}(w)^{1-\psi^{\prime}}=N^{\prime}\left(y^{\psi_{L / K}^{\prime}}\right)^{1-\psi^{\prime}}
$$

(заметим, что $\left.N^{\prime}\left(w_{i}^{\tau_{i}-1}\right)=1, i<k\right)$; тогда для $\zeta \rightleftharpoons N^{\prime}\left(w\left(y^{\psi_{f_{L / K}}^{\prime}}\right)^{-1}\right)$ имеем $\zeta^{1-\psi^{\prime}}=1$, т.е. $\zeta \in M^{\psi^{\prime}} ;$ кроме того, $\zeta \in M_{u}^{K}$; отсюда $\zeta \in M^{\psi^{\prime}} \cap M_{u}^{K}=K$ $\left(\zeta \in U_{K}\right)$. 

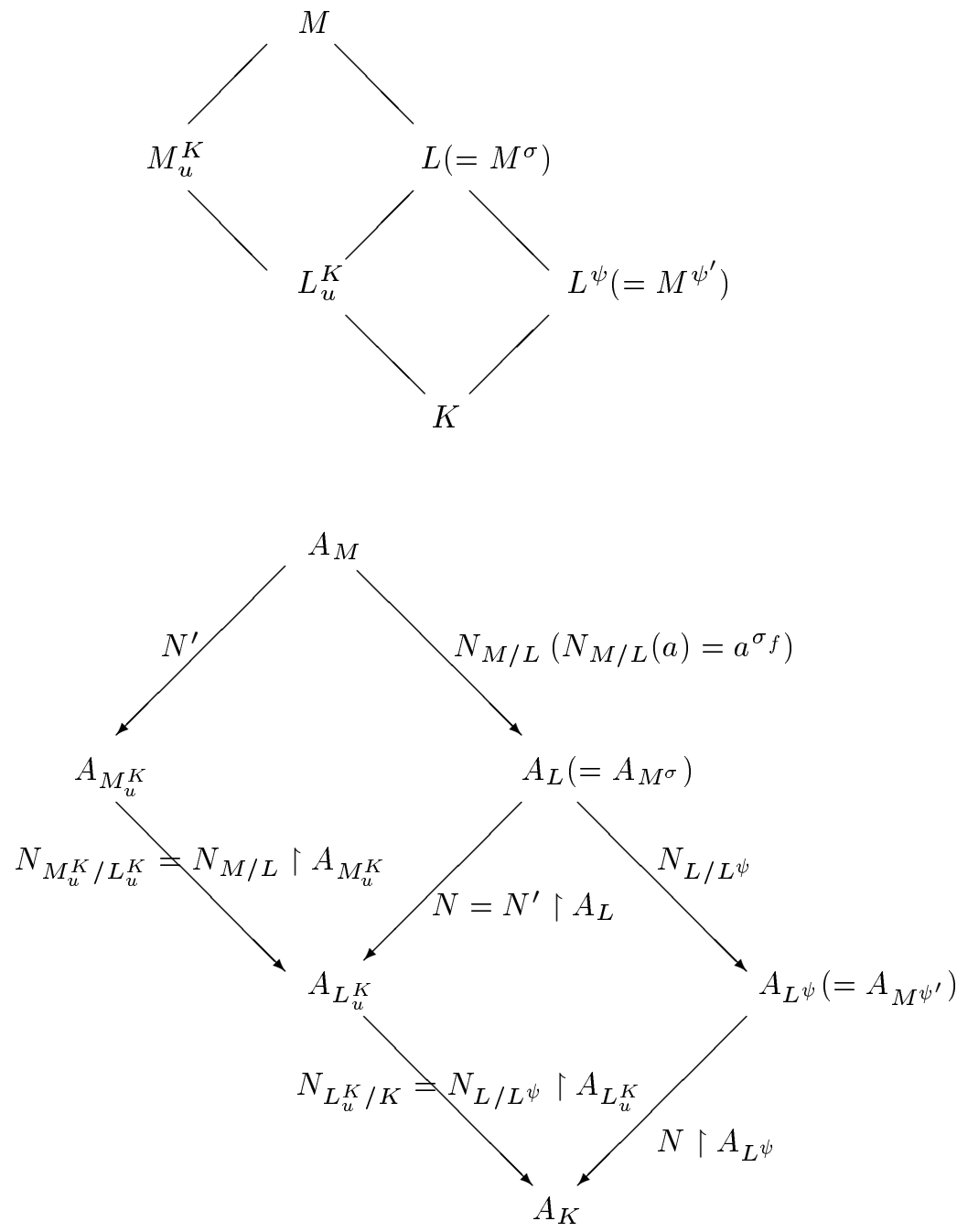

Рис. 4

Итак, $N^{\prime}(w)=\zeta N^{\prime}\left(y^{\psi_{f_{L / K}}^{\prime}}\right)$. Применим норменное отображение $N_{M / L}$ к обеим частям этого равенства.

$$
\begin{aligned}
N_{M / L} N^{\prime}(w) & =N^{\prime}(w)^{\sigma_{f}}=N^{\prime}\left(w^{\sigma_{f}}\right)=N_{M / L_{u}^{K}}(w) \\
& =\zeta^{f} N^{\prime}\left(y^{\psi_{f_{L / K}}^{\prime}}\right)^{\sigma_{f}}=\zeta^{f} N^{\prime}\left(y^{\psi_{f_{L / K}}^{\sigma_{f}}}\right) ;
\end{aligned}
$$

но $\psi_{f_{L / K}}^{\prime}\left(\psi^{\prime} f_{L / K}\right)_{f}=\psi_{f f_{L / K}}^{\prime}=\psi_{f_{M / K}}^{\prime}$ и

$$
N^{\prime}\left(y^{\psi_{f_{M / K}}^{\prime}}\right)=N N_{M / M^{\psi^{\prime}}}(y)=N_{M / K}(y)=N_{L / K}\left(N_{M / K}(y)\right) \in N_{L / K}\left(U_{L}\right) ;
$$

кроме того, имеем

$$
\zeta^{f} \in U_{K}^{f} \leqslant U_{K}^{n} \leqslant N_{L / K}\left(U_{L}\right)
$$


Отсюда $N(v)=N N_{M / L}(w)=N_{M / L_{u}^{K}}(w) \in N_{L / K}\left(U_{L}\right)$.

Предложение доказано.

СлЕДСТВИЕ 2. Гомоморфизм $\rho: U_{K}^{L} \longrightarrow H_{K}^{-1}\left(H, U_{L}\right)$ индуцирует вложение $\bar{\rho}: U_{K}^{L} / N_{L / K}\left(U_{L}\right) \longrightarrow H_{K}^{-1}\left(H, U_{L}\right)$.

Если $M$ - неразветвленное расширение $L$, то нетрудно видеть, что $U_{K}^{L} \leqslant U_{K}^{M}$; $U_{L} \leqslant U_{M}$ индуцирует естественные гомоморфизмы $H^{-1}\left(I_{K}^{L}, U_{L}\right) \longrightarrow H^{-1}\left(I_{K}^{L}, U_{M}\right)$ и $H_{K}^{-1}\left(I_{K}^{L}, U_{L}\right) \longrightarrow H_{K}^{-1}\left(I_{K}^{M}, U_{M}\right)$ и диаграмма

коммутативна.

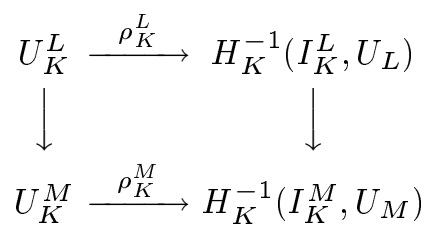

Определим гомоморфизм Хазевинкеля

$$
\bar{\chi}\left(=\bar{\chi}_{K}^{L}\right): H\left(=I_{K}^{L}=G\left(L / L_{u}^{K}\right)\right) \longrightarrow H^{-1}\left(H, U_{L}\right)
$$

так: пусть $\pi_{L}-$ простой элемент $A_{L}$; тогда для $\tau \in H$ полагаем $\bar{\chi}(\tau) \rightleftharpoons \pi_{L}^{\tau-1}$. $I_{G\left(L / L_{u}^{K}\right)} U_{L}$

Проверим корректность этого определения. Из предложения 4 (i) следует, что $v_{L}\left(\pi_{L}^{\tau}\right)=v_{L}\left(\pi_{L}\right)$ и, следовательно, $v_{L}\left(\pi_{L}^{\tau-1}\right)=1, \pi_{L}^{\tau-1} \in U_{L} ;$ кроме того, $N\left(\pi_{L}^{\tau-1}\right)=1$, следовательно, $\pi_{L}^{\tau-1} \in_{N} U_{L}$.

Проверим, что определение $\bar{\chi}$ не зависит от выбора $\pi_{L}$. Пусть $\pi_{L}^{\prime}-$ другой простой элемент $A_{L}$; тогда $\pi_{L}^{\prime}=\pi_{L} u$ для $u\left(=\pi_{L}^{\prime} \pi_{L}^{-1}\right) \in U_{L} ;\left(\pi_{L}^{\prime}\right)^{\tau-1}=\left(\pi_{L} u\right)^{\tau-1}=$ $\left(\pi_{L}\right)^{\tau-1} u^{\tau-1} ;$ но $\left.u^{\tau-1} \in I_{G\left(L / L_{u}^{K}\right.}\right) U_{L}$, следовательно, $\left(\pi_{L}^{\prime}\right)^{\tau-1} \cdot I_{G\left(L / L_{u}^{K}\right)} U_{L}=$ $\pi_{L}^{\tau-1} I_{G\left(L / L_{u}^{K}\right)} U_{L}$.

Проверим, что $\bar{\chi}$ является гомоморфизмом. Пусть $\tau_{0}, \tau_{1} \in H$; тогда $\tau_{0} \tau_{1}-1=$ $\tau_{0} \tau_{1}-\tau_{0}+\tau_{0}-1=\tau_{0}\left(\tau_{1}-1\right)+\tau_{0}-1 ; \pi_{L}^{\tau_{0} \tau_{1}-1}=\left(\pi_{L}^{\tau_{0}}\right)^{\tau_{1}-1} \pi_{L}^{\tau_{0}-1}=\pi_{L}^{\tau_{0}-1}\left(\pi_{L}^{\tau_{0}}\right)^{\tau_{1}-1} ;$ но $\pi_{L}^{\tau_{0}}$ является простым элементом $A_{L}$, поэтому по доказанному выше имеем $\bar{\chi}\left(\tau_{0} \tau_{1}\right)=\bar{\chi}\left(\tau_{0}\right) \cdot \bar{\chi}\left(\tau_{1}\right)$.

Гомоморфизм Хазевинкеля $\bar{\chi}$ индуцирует гомоморфизм $\chi_{*}\left(=\bar{\chi}_{* K}^{L}\right): H \longrightarrow$ $H_{K}^{-1}\left(H, U_{L}\right)$. Из замечания 10 сразу вытекает, что $\chi_{*}\left(\tau^{\psi}\right)=\chi_{*}(\tau)$ для любого элемента $\tau \in H$ (более того, $\chi_{*}\left(\tau^{\sigma}\right)=\chi_{*}(\tau)$ для любых $\tau \in H, \sigma \in G(L / K)$ ).

Итак, имеем два гомоморфизма $\rho: U_{K}^{L} \longrightarrow H_{K}^{-1}\left(H, U_{L}\right)$ и $\chi_{*}: H \longrightarrow H_{K}^{-1}\left(H, U_{L}\right)$.

Пусть $H_{L / K} \rightleftharpoons\left\{\tau \mid \tau \in H, \chi_{*}(\tau) \in \rho\left(U_{L}\right)\right\} ;$ нетрудно видеть, что $H_{L / K}(\leqslant H)-$ нормальная подгруппа $G(L / K)$ и определен естественный гомоморфизм $\chi\left(=\chi_{K}^{L}\right)$ : $H_{L / K} \longrightarrow U_{K}^{L} / N_{L / K}\left(U_{L}\right)$ такой, что диаграмма, изображенная на рис. 5 , коммутативна.

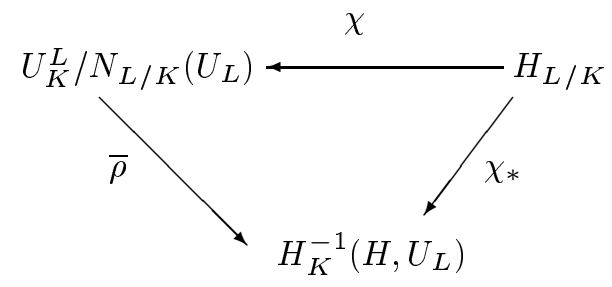

Рис. 5 
Если $M$ - неразветвленное расширение $L$, то $H_{L / K} \leqslant H_{M / K}$ и коммутативна диаграмма, изображенная на рис. 6.

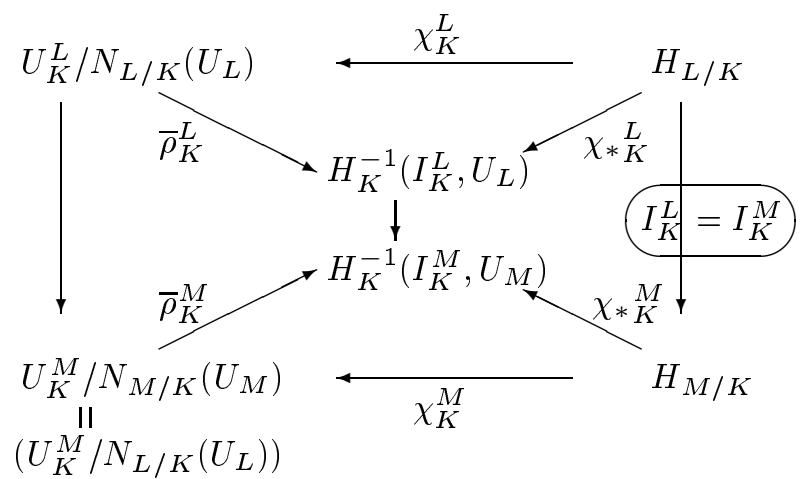

Рис. 6

Отображение $\chi$ можно использовать для вычисления отображения Нейкирха.

Лемма 8. Пусть $\psi \in \operatorname{Frob}(L / K), \partial_{K}^{L} \psi=1, \tau \in H u \psi \tau \in \operatorname{Frob}(L / K)(u$, следовательно, $\left.\partial_{K}^{L}(\psi \tau)=1\right)$, тогда $\tau \in H_{L / K} u$

$$
\nu(\psi \tau)=\nu(\psi) \chi(\tau)
$$

Заметим, что $U_{L}^{K} / N_{L / K}\left(U_{L}\right)$ является подгруппой $A_{K} / N_{L / K}\left(U_{L}\right)$.

ДоказАТЕЛЬство. Пусть $\pi_{\psi}-$ простой элемент $A_{L \psi}, \pi_{\psi \tau}-$ простой элемент $A_{L \psi \tau}$. Так как $L$ является непосредственньм расширением как $L^{\psi}$, так и $L^{\psi \tau}$, то $\pi_{\psi \tau}$ и $\pi_{\psi}$ являются простыми элементами $A_{L}$. Тогда $\pi_{\psi \tau}=\pi_{\psi} u$ для $u\left(=\pi_{\psi \tau} \pi_{\psi}^{-1}\right) \in U_{L} . \quad$ Так как $\pi_{\psi}^{\psi}=\pi_{\psi}, \pi_{\psi \tau}^{\psi \tau}=\pi_{\psi \tau}$, то имеем $\pi_{\psi} u=\pi_{\psi \tau}=\pi_{\psi \tau}^{\psi \tau}=\left(\pi_{\psi} u\right)^{\psi \tau}=\pi_{\psi}^{\psi \tau} u^{\psi \tau}=\pi_{\psi}^{\tau} u^{\psi \tau}$ и $u^{1-\psi \tau}=\pi_{\psi}^{\tau-1}$. Проверим, что $v \rightleftharpoons N(u)=N_{L / L_{u}^{K}}(u) \in U_{K}$. Так как $\pi_{\psi} \in A_{L}, N\left(\pi_{\psi}\right) \in A_{L_{u}^{K}}$, то $N\left(\pi_{\psi}\right) \in A_{L_{u}^{K} \cap L \psi}=A_{K}$; аналогично, $N\left(\pi_{\psi \tau}\right) \in A_{K} ;$ но тогда и $N(u)=$ $N\left(\pi_{\psi \tau}\right) N\left(\pi_{\psi}\right)^{-1} \in A_{K} ;$ и $u \in U_{L}$ влечет, что $N(u) \in U_{K}$. Итак, $v=N(u) \in U_{K}^{L}$ и $\rho(v)=u^{1-\psi \tau} \cdot V_{K}\left(L / L_{u}^{K}\right)=\pi_{\psi}^{\tau-1} \cdot V_{K}\left(L / L_{u}^{K}\right)=\bar{\chi}(\tau) . \quad$ Тогда $\tau \in H_{L / K}$, $\chi_{*}(\tau)=v N_{L / K}\left(U_{L}\right)$ и имеем $N_{L \psi \tau / K}\left(\pi_{\psi \tau}\right)=N\left(\pi_{\psi \tau}\right)=N\left(\pi_{\psi} u\right)=N(\pi \psi)$. $N(u)=N_{L \psi / K}\left(\pi_{\psi}\right) \cdot v$, отсюда и получаем нужное соотношение $\nu(\psi \tau)=\nu(\psi) \cdot$ $\chi(\tau)$.

СЛЕДСТВИЕ 3. Если $M \geqslant L$ - неразветвленное расширение L, удовлетворяющее предложсению 3 , то $H_{M / K}=I_{K}^{M}\left(=I_{K}^{L}\right)$.

Гомоморфизм $\chi_{K}^{M}: H=I_{K}^{L} \longrightarrow U_{K}^{M} / N_{M / K}\left(U_{M}\right) \leqslant A_{K} / N_{L / K}\left(U_{L}\right)$ не зависит от выбора такого $M$; его будем обозначать $\chi^{L}$.

Пусть $\psi^{\prime} \in \operatorname{Frob}(L / K), m=\partial_{K}^{L} \psi^{\prime}$; тогда $\psi^{\prime}=\psi^{m} \tau$ для подходящего $\tau(\rightleftharpoons$ $\left.\psi^{-m} \psi^{\prime}\right) \in H=I_{K}^{L}$ (здесь по-прежнему $\psi$ обозначает элемент из $\operatorname{Frob}(L / K)$ с 


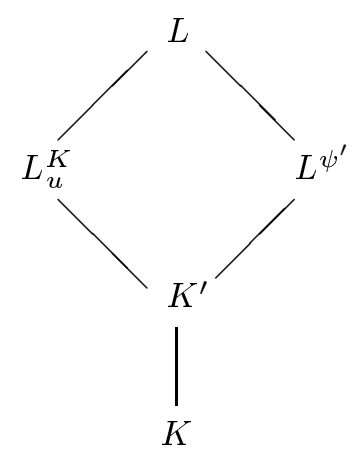

Рис. 7

условием $\left.\partial_{K}^{L} \psi=1\right)$. Пусть $K^{\prime} \rightleftharpoons\left(L_{u}^{K}\right)^{\psi^{\prime}}$; тогда $\psi^{\prime} \in \operatorname{Frob}\left(L / K^{\prime}\right)$ и $\partial_{K^{\prime}}^{L} \psi^{\prime}=1$; $G\left(K^{\prime} / K\right)=\left\langle\psi\left\lceil K^{\prime}\right\rangle ;\left[K^{\prime}: K\right]=m\right.$ и $N_{K^{\prime} / K}(a)=a^{\psi_{m}}$ для $a \in A_{K^{\prime}}$.

Проверим, что $N_{K^{\prime} / K}\left(U_{K^{\prime}}^{L}\right) \leqslant U_{K}^{L}$. Пусть $u^{\prime} \in U_{K^{\prime}}^{L}, u \rightleftharpoons N_{K^{\prime} / K}\left(u^{\prime}\right)$ и $v^{\prime} \in U_{L}$ такой, что $N\left(v^{\prime}\right)=u^{\prime}$; пусть $v \rightleftharpoons v^{\prime \psi_{m}} \in U_{L}$; тогда $N(v)=N\left(v^{\prime} \psi_{m}\right)=N\left(v^{\prime}\right)^{\psi_{m}}=$ $u^{\prime \psi_{m}}=N_{K^{\prime} / K}\left(u^{\prime}\right)=u$; следовательно, $u=N\left(u^{\prime}\right) \in U_{K}^{L}$.

Имеем $H=I_{K}^{L}=I_{K^{\prime}}^{L}$; проверим, что $V_{K^{\prime}}\left(L / L_{u}^{K}\right) \leqslant V_{K}\left(L / L_{u}^{K}\right)$; для этого достаточно показать, что для $w \in_{N} U_{L}$ элемент $w^{1-\psi^{\prime}}$ принадлежит $V_{K}\left(L / L_{u}^{K}\right)$; но имеют место следующие равенства и сравнения:

$w^{1-\psi^{\prime}}=w^{1-\psi^{m} \tau}=w^{1-\psi^{m}}\left(w^{\psi^{m}}\right)^{1-\tau} \equiv w^{1-\psi^{m}} \equiv\left(w^{1-\psi}\right)^{m} \quad \bmod I_{G\left(L / L_{u}^{K}\right)} U_{L}$.

Вложение $V_{K^{\prime}}\left(L / L_{u}^{K}\right) \leqslant V_{K}\left(L / L_{u}^{K}\right)$ индуцирует эпиморфизм $\varepsilon: H_{K^{\prime}}^{-1}\left(H, U_{L}\right) \longrightarrow$ $H_{K}^{-1}\left(H, U_{L}\right)$.

Проверим, что следующая диаграмма

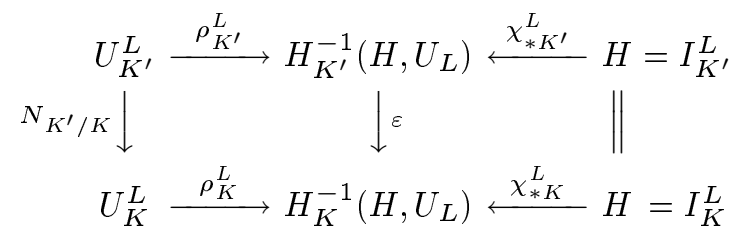

коммутативна.

Пусть $u^{\prime} \in U_{K^{\prime}}^{L}, v^{\prime} \in U_{L}$ такой, что $N\left(v^{\prime}\right)=u^{\prime}$. Выше было показано, что для $v \rightleftharpoons v^{\prime} \psi_{m} \in U_{L}$ имеем $N(v)=u=N_{K^{\prime} / K}\left(u^{\prime}\right)$. Далее,

$$
\rho_{K^{\prime}}^{L}\left(u^{\prime}\right)=\left(v^{\prime}\right)^{1-\psi^{\prime}} V_{K^{\prime}}\left(L / L_{u}^{K}\right)
$$

и, как проверено выше,

$$
\left(v^{\prime}\right)^{1-\psi^{\prime}} \equiv\left(v^{\prime}\right)^{1-\psi^{m}} \quad \bmod V_{K^{\prime}}\left(L / L_{u}^{K}\right) ; \quad\left(v^{\prime}\right)^{1-\psi^{m}}=\left(\left(v^{\prime}\right)^{\psi_{m}}\right)^{1-\psi}=v^{1-\psi} .
$$

Отсюда и следует, что $\varepsilon \rho_{K^{\prime}}^{L}\left(u^{\prime}\right)=\rho_{K}^{L} N_{K^{\prime} / K}\left(u^{\prime}\right)$. Итак, проверена коммутативность левого квадрата. Коммутативность правого квадрата очевидна. 
Коммутативность диаграммы (**) влечет вложение $H_{L / K^{\prime}} \leqslant H_{L / K}$ и коммутативность диаграммы

$$
\begin{array}{cc}
H_{L / K^{\prime}} \stackrel{\chi_{K^{\prime}}^{L}}{\longrightarrow} U_{K^{\prime}}^{L} / N_{L / K^{\prime}}\left(U_{L}\right) \\
\downarrow & \downarrow \widetilde{N}_{K^{\prime} / K} \\
H_{L / K} \stackrel{\chi_{K}^{L}}{\longrightarrow} U_{K}^{L} / N_{L / K}\left(U_{L}\right) .
\end{array}
$$

СлеДСтвИЕ 4. Пусть $\psi^{\prime} \in \operatorname{Frob}(L / K), \tau \in H, \psi^{\prime} \tau \in \operatorname{Frob}(L / K)$, mогдa $\tau \in H_{L / K} u$

$$
\nu_{L / K}\left(\psi^{\prime} \tau\right)=\nu_{L / K}\left(\psi^{\prime}\right) \cdot \chi^{L}(\tau)
$$

ДоКАЗАТЕЛьство. Действительно, по лемме 8 имеем

$$
\nu_{L / K^{\prime}}\left(\psi^{\prime} \tau\right)=\nu_{L / K^{\prime}}\left(\psi^{\prime}\right) \cdot \chi_{K^{\prime}}^{L}(\tau)
$$

а по лемме 5 имеем

$$
\begin{aligned}
\nu_{L / K}\left(\psi^{\prime} \tau\right) & =\widetilde{N}_{K^{\prime} / K}\left(\nu_{L / K^{\prime}}\left(\psi^{\prime} \tau\right)\right)=\widetilde{N}_{K^{\prime} / K}\left(\nu_{L / K^{\prime}}\left(\psi^{\prime}\right) \cdot \chi_{K^{\prime}}^{L}(\tau)\right) \\
& =\widetilde{N}_{K^{\prime} / K}\left(\nu_{L / K^{\prime}}\left(\psi^{\prime}\right)\right) \cdot \widetilde{N}_{K^{\prime} / K} \chi_{K^{\prime}}^{L}(\tau)=\nu_{L / K}(\psi) \cdot \chi_{K^{\prime}}^{L}(\tau)
\end{aligned}
$$

ПРЕДЛОЖЕНИЕ 7. Если $\psi_{0}, \psi_{1} \in \operatorname{Frob}(L / K)$ когерентны, то $\nu_{L / K}\left(\psi_{0} \psi_{1}\right)=$ $\nu_{L / K}\left(\psi_{0}\right) \cdot \nu_{L / K}\left(\psi_{1}\right)$.

ДокАЗАТЕльство. Пусть $m_{0} \rightleftharpoons \partial_{K}^{L} \psi_{0}, m_{1} \rightleftharpoons \partial_{K}^{L} \psi_{1}$ и $\tau_{0}, \tau_{1} \in H_{L / K}$ таковы, что $\psi_{0}=\psi^{m_{0}} \tau_{0}, \psi_{1}=\psi^{m_{1}} \tau_{1}$; тогда $\psi_{2} \rightleftharpoons \psi_{0} \psi_{1}=\psi^{m_{2}} \tau_{2}, m_{2}=m_{0}+m_{1}$, $\tau_{2}=\tau_{0}^{\psi^{m_{1}}} \tau_{1} \in H_{L / K}$. Имеем (используя леммы 5,7 и следствие 4 )

$$
\begin{gathered}
\nu_{L / K}\left(\psi_{2}\right)=\nu_{L / K}\left(\psi^{m_{2}} \tau_{2}\right)=\nu_{L / K}\left(\psi^{m_{2}}\right) \cdot \chi_{K}^{L}\left(\tau_{2}\right) \\
\nu_{L / K}\left(\psi^{m_{2}}\right)=\nu_{L / K}(\psi)^{m_{0}+m_{1}}=\nu_{L / K}(\psi)^{m_{0}} \cdot \nu_{L / K}(\psi)^{m_{1}} \\
=\nu_{L / K}\left(\psi^{m_{0}}\right) \cdot \nu_{L / K}\left(\psi^{m_{1}}\right) ; \\
\chi_{* K}^{L}\left(\tau_{2}\right)=\chi_{* K}^{L}\left(\tau_{0}^{\psi^{m_{1}}} \tau_{1}\right)=\chi_{* K}^{L}\left(\tau_{0}^{\psi^{m_{1}}}\right) \cdot \chi_{* K}^{L}\left(\tau_{1}\right)=\chi_{* K}^{L}\left(\tau_{0}\right) \cdot \chi_{* K}^{L}\left(\tau_{1}\right) .
\end{gathered}
$$

Итак,

$$
\begin{aligned}
\nu_{L / K}\left(\psi_{0}\right) & =\nu_{L / K}\left(\psi^{m_{0}}\right) \cdot \chi_{* K}^{L}\left(\tau_{0}\right) \cdot \nu_{L / K}\left(\psi^{m_{1}}\right) \cdot \chi_{* K}^{L}\left(\tau_{1}\right) \\
& =\nu_{L / K}\left(\psi^{m_{0}} \tau_{0}\right) \cdot \nu_{L / K}\left(\psi^{m_{1}} \tau_{1}\right)=\nu_{L / K}\left(\psi_{0}\right) \cdot \nu_{L / K}\left(\psi_{1}\right)
\end{aligned}
$$

Определим теперь гомоморфизм взаимности

$$
r_{L / K}: G(L / K) \longrightarrow A_{K} / N_{L / K}\left(A_{L}\right)
$$

следуюшим образом: пусть $\sigma \in G(L / K), M \geqslant L$ - неразветвленное расширение $L$ такое, что для $\sigma$ сушествует подъем $\psi \in \operatorname{Frob}(M / K)$, тогда

$$
r_{L / K}(\sigma) \rightleftharpoons \nu_{M / K}(\psi) \cdot N_{L / K}\left(A_{L}\right)
$$


Отметим, что $\nu_{M / K}(\psi) \in A_{K} / N_{M / K}\left(U_{M}\right)$ и $N_{M / K}\left(U_{M}\right) \leqslant N_{L / K}\left(U_{L}\right) \leqslant$ $N_{L / K}\left(A_{L}\right)$.

Из предложения 3 следует, что для любого $\sigma \in G(L / K)$ найдется неразветвленное расширение $M \geqslant L$ такое, что $\sigma$ имеет подъем в $\operatorname{Frob}(M / K)$.

Проверим корректность определения отображения $r_{L / K}$. Пусть $M^{\prime} \geqslant M \geqslant L-$ неразветвленные расширения $L, \psi^{\prime} \in \operatorname{Frob}\left(M^{\prime} / K\right)$ и $\psi \in \operatorname{Frob}(M / K)$ - подъемы $\sigma$. Тогда по лемме 2 имеем $\psi^{\prime} \uparrow M=\psi$ и по замечанию $8 \nu_{M^{\prime} / K}\left(\psi^{\prime}\right)=\nu_{M / K}(\psi)$. Заметим, что если $M_{0} \geqslant L, M_{1} \geqslant L$ - неразветвленные расширения $L$ такие, что $\sigma$ имеет подъемы $\psi_{i}$ в $\operatorname{Frob}\left(M_{i} / K\right), i=0,1$, то по предложению 3 найдется неразветвленное расширение $M \geqslant L$ такое, что $M \geqslant M_{i}, i=0,1$, и $\sigma$ имеет подъем $\psi$ в $\operatorname{Frob}(M / K)$. Тогда по установленному выше $\nu_{M / K}(\psi)=\nu_{M_{i} / K}\left(\psi_{i}\right)$, $i=0,1$. Корректность определения $r_{L / K}$ проверена.

ПРЕДЛОЖЕНИЕ 8. Отображсение $r_{L / K}: G(L / K) \longrightarrow A_{K} / N_{L / K}\left(A_{L}\right)$ явяяется гомоморфизмом.

ДокАЗАтЕльство. Пусть $M$-неразветвленное расширение $L$, удовлетворяющее предложению 3 . Пусть $\sigma_{0}, \sigma_{1} \in G(L / K), \sigma_{2} \rightleftharpoons \sigma_{0} \sigma_{1}$ и $\psi_{0}, \psi_{1}, \psi_{2} \in \operatorname{Frob}(M / K)$ - подъемы элементов $\sigma_{0}, \sigma_{1}$ и $\sigma_{2}$ соответственно. Сравним элементы $\psi_{0} \psi_{1} \in$ $\operatorname{Frob}(M / K)$ и $\psi_{2}$. Имеем

$$
\begin{gathered}
\left(\psi_{0} \psi_{1}\right) \uparrow L=\left(\psi_{0} \uparrow L\right)\left(\psi_{1} \uparrow L\right)=\sigma_{0} \sigma_{1}=\sigma_{2} ; \quad \psi_{2} \uparrow L=\sigma_{2} \\
\partial_{L}^{M}\left(\psi_{0} \psi_{1}\right)=\partial_{K}^{M}\left(\psi_{0}\right)+\partial_{K}^{M}\left(\psi_{1}\right)
\end{gathered}
$$

(так как $\psi_{0}, \psi_{1}$ когерентны); $\partial_{K}^{M}\left(\psi_{0}\right)=\partial_{K}^{L}\left(\sigma_{0}\right) \leqslant f_{L / K} ; \partial_{K}^{M}\left(\psi_{1}\right)=\partial_{K}^{L}\left(\sigma_{1}\right) \leqslant$ $f_{L / K} ;$ следовательно, $\partial_{K}^{M}\left(\psi_{0} \psi_{1}\right) \leqslant 2 f_{L / K}$. Из определения $\partial$ следует, что $\left(\psi_{0} \psi_{1}\right) \uparrow M_{u}^{K}=\left(\Phi_{K}^{M}\right)^{\partial_{K}^{M}\left(\psi_{0} \psi_{1}\right)} ;\left(\psi_{0} \psi_{1}\right) \uparrow L_{u}^{K}=\left(\Phi_{K}^{M} \uparrow L_{u}^{K}\right)^{\partial_{K}^{M}\left(\psi_{0} \psi_{1}\right)} ;$ но $\left(\psi_{0} \psi_{1}\right) \uparrow L_{u}^{K}=\sigma_{2} \uparrow L_{u}^{K}=\left(\Phi_{K}^{L}\right) \partial_{K}^{L}\left(\sigma_{2}\right)\left(=\partial_{K}^{M}\left(\psi_{2}\right)\right)$; так как $\left|\Phi_{K}^{L}\right|=f_{L / K}$, имеет место сравнение $\partial_{K}^{M}\left(\psi_{0} \psi_{1}\right) \equiv \partial_{K}^{M}\left(\psi_{2}\right) \bmod f_{L / K}$. Это сравнение вместе с соотношениями

$$
0<\partial_{K}^{M}\left(\psi_{0} \psi_{1}\right) \leqslant 2 f_{L / K}, \quad 0<\partial_{K}^{M}\left(\psi_{2}\right) \leqslant f_{L / K}
$$

показывает, что возможны два случая: $\partial_{K}^{M}\left(\psi_{0} \psi_{1}\right)=\partial_{K}^{M}\left(\psi_{2}\right)=\partial_{K}^{L}\left(\sigma_{2}\right)$ (и тогда $\psi_{0} \psi_{1}$ является подъемом $\sigma_{2}$ и $\left.\psi_{2}=\psi_{0} \psi_{1}\right)$ и $\partial_{K}^{M}\left(\psi_{0} \psi_{1}\right)=\partial_{K}^{M}\left(\psi_{2}\right)+f_{L / K}=$ $\partial_{K}^{L}\left(\sigma_{2}\right)+f_{L / K}$. В случае $\psi_{2}=\psi_{0} \psi_{1}$ имеем

$$
\begin{aligned}
r_{L / K}\left(\sigma_{2}\right) & =\nu_{M / L}\left(\psi_{2}\right) \cdot N_{L / K}\left(A_{L}\right)=\nu_{M / L}\left(\psi_{0} \psi_{1}\right) \cdot N_{L / K}\left(A_{L}\right) \\
& =\nu_{M / L}\left(\psi_{0}\right) \cdot \nu_{M / L}\left(\psi_{1}\right) \cdot N_{L / K}\left(A_{L}\right)=r_{L / K}\left(\sigma_{0}\right) \cdot r_{L / K}\left(\sigma_{1}\right) .
\end{aligned}
$$

В случае, когда

$$
\partial_{K}^{M}\left(\psi_{0} \psi_{1}\right)=\partial_{K}^{M}\left(\psi_{2}\right)+f_{L / K}
$$

пусть $\psi$ - подъем единицы в $\operatorname{Frob}(M / K)$; тогда $\psi \uparrow L=\operatorname{id}_{L}, \partial_{K}^{M} \psi=\partial_{K}^{M} 1=f_{L / K}$. Рассмотрим элементы $\psi_{0} \psi_{1}, \psi \psi_{2} \in \operatorname{Frob}(M / K)$; имеем $\partial_{K}^{M}\left(\psi_{0} \psi_{1}\right)=f_{L / K}+$ $\partial_{K}^{M}\left(\psi_{0} \psi_{2}\right)=\partial_{K}^{M}\left(\psi \psi_{2}\right)$; вместе с $\left(\psi_{0} \psi_{1}\right) \uparrow L=\sigma_{2}=\left(\psi \psi_{2}\right) \uparrow L$ и замечанием 5 это дает равенство $\psi_{0} \psi_{1}=\psi \psi_{2}$ и

$$
\nu_{M / K}\left(\psi_{0} \psi_{1}\right)=\nu_{M / K}\left(\psi_{0}\right) \cdot \nu_{M / K}\left(\psi_{1}\right)=\nu_{M / K}\left(\psi \psi_{2}\right)=\nu_{M / K}(\psi) \cdot \nu_{M / K}\left(\psi_{2}\right)
$$


Для завершения доказательства предложения достаточно установить, что $\nu_{M / K}(\psi) \in N_{L / K}\left(A_{L}\right) / N_{L / K}\left(U_{L}\right)$. Напомним, что $\psi$ - подъем единицы, следовательно, $\psi \uparrow L=\operatorname{id}_{L} ; L \leqslant M^{\psi} ;$ так как $\psi \in \operatorname{Frob}(M / K)$, имеем $|\psi| \cdot \partial_{K}^{M} \psi=f_{M / K}\left(=f_{M / L} \cdot f_{L / K}\right) ;$ но $\partial_{K}^{M} \psi=\partial_{k}^{L} 1=f_{L / K}$, следовательно, $|\psi|=f_{M / L}$ и $\left[M: M^{\psi}\right]=|\psi|=f_{M / L}=[M: L]$, поскольку $M$ - неразветвленное расширение $L$. Пусть $\pi$-простой элемент $A_{M \psi}=A_{L}$, тогда $\nu_{M / K}(\psi)=N_{L / K}(\pi)$. $N_{L / K}\left(U_{L}\right)$, что и требовалось.

СлЕДСТВИЕ 5. Для $\tau \in H=I_{K}^{L} \leqslant G(L / K)$ имеет место соотношение

$$
r_{L / K}(\tau)=\chi^{L}(\tau) N_{L / K}\left(A_{L}\right)
$$

ДокАЗАТЕЛьство. Действительно, если $M \geqslant L$ выбрано как в доказательстве предложения $8, \psi \in \operatorname{Frob}(M / K)$ - подъем единищы, то легко видеть, что $\psi \tau-$ подъем $\tau\left(\psi \tau \uparrow L=(\psi \uparrow L)(\tau \uparrow L)=1 \cdot \tau=\tau ; \partial_{K}^{M} \psi \tau=\partial_{K}^{M} \psi=f_{L / K}=\partial_{K}^{M} \tau\right.$ и $\psi \tau \in \operatorname{Frob}(M / K))$. Тогда $\nu_{M / K}(\psi \tau)=\nu_{M / K}(\psi) \cdot \chi_{K}^{M}(\tau)$ по следствию 4 и $r_{L / K}(\tau)=\nu_{M / K}(\psi \tau) \cdot N_{L / K}\left(A_{L}\right)=\nu_{M / K}(\psi) \cdot \chi_{K}^{M}(\tau) \cdot N_{L / K}\left(A_{L}\right)=\chi^{L}(\tau) \cdot$ $N_{L / K}\left(A_{L}\right)$, так как $\nu_{M / K}(\psi) \in N_{L / K}\left(A_{L}\right)$.

Используя идеи доказательства теоремы (6.3) из [2], установим, что гомоморфизм взаимности $r_{L / K}$ индуцирует вложение $r_{L / K}: G(L / K)^{\mathrm{ab}} \longrightarrow$ $A_{K} / N_{L / K}\left(A_{L}\right)$ (теорема 1) и если наложить дополнительное условие (аксиома $R$ ), то вложение $r_{L / K}: G(L / K)^{\text {ab }} \longrightarrow A_{K} / N_{L / K}\left(A_{L}\right)$ будет изоморфизмом (теорема 2).

Теорема 1. Пусть $L \geqslant K$ - конечное расширение Галуа, тогда гомомор$\oint$ изм взаимности $r_{L / K}: G(L / K) \longrightarrow A_{K} / N_{L / K}\left(A_{L}\right)$ индуцирует вложсение (которое будем обозначать так жсе)

$$
r_{L / K}: G(L / K)^{\mathrm{ab}} \longrightarrow A_{K} / N_{L / K}\left(A_{L}\right)
$$

Здесь через $G^{\mathrm{ab}}$ обозначается факторгруппа $G / G^{\prime}$ группь $G$ по коммутанmy $G^{\prime}\left(G^{\mathrm{ab}}\right.$ - наибольиая абелева факторгруппа $\left.G\right)$.

Установим следующую лемму.

ЛЕмма 9. Пусть $L$ - конечное расиирение $K, M$ - промехуточное поле $(L \geqslant M \geqslant K)$. Тогда коммутативна следующая диаграмма:

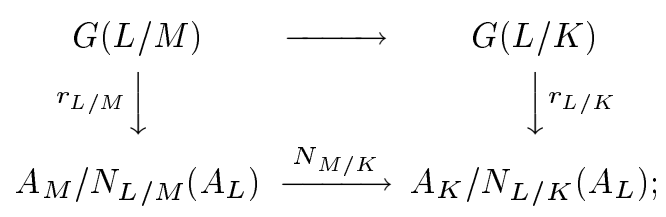

если еще $M$ - расширение Галуа поля $K$, то коммутативна диаграмма

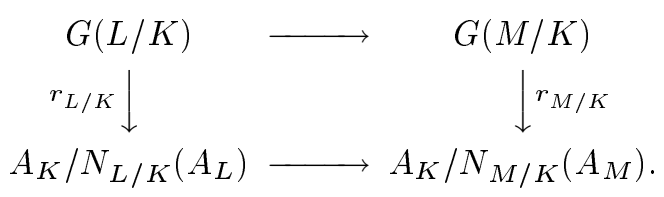


Здесь верхняя стрелка первой диаграммы - это вложение $G(L / M) \leqslant G(L / K)$; нижняя стрелка этой диаграммы индуцирована гомоморфизмом $N_{M / K}: A_{M} \longrightarrow$ $A_{K}$ (так как $\left.N_{M / K} N_{L / M}\left(A_{L}\right)=N_{L / K}\left(A_{L}\right)\right)$; верхняя стрелка второй диаграммы - это отображение ограничения $\sigma \longmapsto \sigma \uparrow M, \sigma \in G(L / K)$; нижняя стрелка этой диаграммы индуцирована вложением $N_{L / K}\left(A_{L}\right) \leqslant N_{M / K}\left(A_{M}\right)$ (так как $\left.N_{L / K}\left(A_{L}\right)=N_{M / K} N_{L / M}\left(A_{L}\right)\right)$.

Коммутативность первой диаграммы следует из определения гомоморфизма взаимности и следующего утверждения (обобщающего лемму 5): для $\psi \in \operatorname{Frob}(L / M)$ $(\subseteq \operatorname{Frob}(L / K))$ имеет место соотношение $N_{M / K} \nu_{L / M}(\psi)=\nu_{L / K}(\psi)$.

Доказательство дословно совпадает с доказательством леммы 5.

Коммутативность второй диаграммы следует из определения гомоморфизма взаимности и предложения 5.

Пусть $K \leqslant M \leqslant L ; L, M$ - конечные расширения Галуа поля $K$. Тогда из леммы 9 следует, что диаграмма $(+)$ коммутативна:

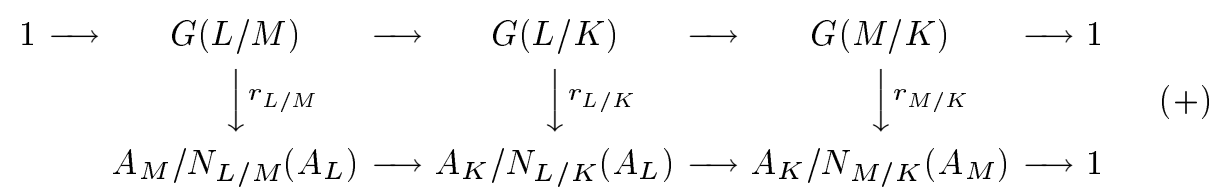

(горизонтальные последовательности в $(+)$ точны).

СлеДСТВИЕ 6. Если $r_{L / K}: G(L / K)^{\mathrm{ab}} \longrightarrow A_{K} / N_{L / K}\left(A_{L}\right)-$ вложение, $r_{L / M}: G(L / M) \longrightarrow A_{M} / N_{L / M}\left(A_{L}\right)$ - эпиморфизм, то $r_{M / K}: G(M / K)^{\mathrm{ab}} \longrightarrow$ $A_{L} / N_{M / L}\left(A_{M}\right)$ - вложение.

ДОКАЗАТЕЛЬСТво ТЕОРЕмЫ 1 . Пусть $L_{\mathrm{ab}}$ - наибольшее абелево подрасширение $K$ в $L$; тогда $G\left(L / L_{\mathrm{ab}}\right)=G(L / K)^{\prime}$ - коммутатор группы $G(L / K)$ и $G\left(L_{\mathrm{ab}} / K\right)$ $\simeq G(L / K)^{\mathrm{ab}}$. Полагая $M=L_{\mathrm{ab}}$ в диаграмме $(+)$, легко получим, что если

$$
r_{L_{\mathrm{ab}} / K}: G\left(L_{\mathrm{ab}} / K\right) \longrightarrow A_{K} / N_{L_{\mathrm{ab}} / K}\left(A_{L_{\mathrm{ab}}}\right)
$$

- вложение, то и $r_{L / K}: G(L / K)^{\mathrm{ab}} \longrightarrow A_{K} / N_{L / K}\left(A_{L}\right)$ - вложение.

Таким образом, достаточно доказать теорему для случая, когда $L$ - абелево расширение $K$. Рассмотрим случай, когда $L$ является композитом циклических подрасширений $L \geqslant M_{0}, \ldots, M_{n} \geqslant K$ таких, что для любого $i \leqslant n M_{i}$ либо неразветвлено над $K$, либо вполне разветвлено. Заметим, что тогда $G(L / K)$ естественно вкладывается в $\prod_{i \leqslant n} G\left(M_{i} / K\right)$, и диаграмма $(+)$ позволяет свести доказательство к двум случаям:

1) $L$ - циклическое неразветвленное расширение $K$;

2) $L$ - циклическое вполне разветвленное расширение $K$.

ЛЕмма 10. Пусть $L$ - ииклическое неразветвленное расширение $K$, тогда $r_{L / K}$ является изоморфизмом групп $G(L / K)$ u $A_{K} / N_{L / K}\left(A_{L}\right)$.

ДоКАЗАТЕЛЬСтво ЛЕммы 10. Рассмотрим точную последовательность

$$
1 \longrightarrow U_{K} \longrightarrow A_{K} \stackrel{v_{K}}{\longrightarrow} Z \longrightarrow 1
$$


“ракторизуя” ее по $N_{L / K}\left(A_{L}\right)$, получим точную последовательность

$$
1 \longrightarrow U_{K} / U_{K} \cap N_{L / K}\left(A_{L}\right) \longrightarrow A_{K} / N_{L / K}\left(A_{L}\right) \longrightarrow Z / v_{K}\left(N_{L / K}\left(A_{L}\right)\right) \longrightarrow 1
$$

Нетрудно видеть, что $U_{K} \cap N_{L / K}\left(A_{L}\right)=N_{L / K}\left(U_{L}\right)$; но аксиома $U$ для неразветвленных расширений влечет, что $N_{L / K}\left(U_{L}\right)=U_{K}$; и тогда группа $A_{K} / N_{L / K}\left(A_{L}\right)$ изоморфна группе $Z / v_{K}\left(N_{L / K}\left(A_{L}\right)\right)$.

Далее, неразветвленность $L \geqslant K$ влечет, что $n=[L: K]=f_{L / K}$; из того, что $v\left(N_{L / K}\left(A_{L}\right)\right)=f_{L / K} Z=n Z$, следует, что $Z / v_{K}\left(N_{L / K}\left(A_{L}\right)\right)$ - циклическая группа порядка $n=f_{L / K}$.

Итак, $A_{K} / N_{L / K}\left(A_{L}\right)$ - циклическая группа порядка $n$; ее образуюшей является $\pi N_{L / K}\left(A_{L}\right)$, где $\pi$-простой элемент $A_{K}$.

Элемент $\Phi \uparrow L$ является образующим группы $G(L / K)$. Вычислим $r_{L / K}(\Phi \nmid L)$. Так как $L^{\Phi \nmid L}=K$, то $r_{L / K}(\Phi \uparrow L)=\pi N_{L / K}\left(A_{L}\right)$ и, следовательно, $r_{L / K}$ отображает $G(L / K)$ на $A_{K} / N_{L / K}\left(A_{L}\right)$; так как $n=|G(L / K)|=\left|A_{K} / N_{L / K}\left(A_{L}\right)\right|$, то $r_{L / K}-$ изоморфизм.

ЛЕмма 11. Пусть $L-$ ииклическое вполне разветвленное расиирение $K$, mогда

$$
r_{L / K}: G(L / K) \longrightarrow A_{K} / N_{L / K}\left(A_{L}\right)
$$

является вложсением.

ДокАЗАТЕЛЬСтво ЛЕммы 11. Пусть $L^{\prime}$ - конечное неразветвленное расширение $L$ такое, что $n \rightleftharpoons[L: K]$ делит $\left[L^{\prime}: L\right]$; тогда $H \rightleftharpoons H_{L^{\prime} / K}=H_{L / K}=G$ $(=G(L / K))$ (по лемме 8) и $H_{K}^{-1}\left(H, U_{L}\right)=H^{-1}\left(H, U_{L}\right), H_{K}^{-1}\left(H, U_{L^{\prime}}\right)=$ $H^{-1}\left(H, U_{L^{\prime}}\right)$, так как $L^{\prime}$ - абелево расширение $K$.

Проверим, что гомоморфизм Хазевинкеля $\bar{\chi}: H(=G) \longrightarrow H^{-1}\left(H, U_{L}\right)$ является вложением.

Пусть $\pi_{L}$ - простой элемент $A_{L}, \tau$ - образуюшая группы $G, G=\langle\tau\rangle$. Заметим, что $v_{L}\left(A_{K}\right)=n Z\left(n=[L: K]=e_{L / K}\right)$. Так как $f_{L / K}=1$, то по предложению 4 (ii) имеем для $a \in A_{K} \leqslant A_{L} v_{L}(a)=v_{K}\left(N_{L / K}(a)\right)=v_{K}\left(a^{n}\right)=n v_{K}(a)$, и так как $v_{K}\left(A_{K}\right)=Z$, то $v_{L}\left(A_{K}\right)=n Z$.

Пусть $k \in \omega$ таково, что $\bar{\chi}\left(\tau^{k}\right) \in I_{G} U_{L}=U_{L}^{\tau-1}$, т.е. $\pi_{L}^{\tau^{k}-1}=\left(\pi_{L}^{\tau_{k}}\right)^{\tau-1}=u^{\tau-1}$ для подходящего $u \in U_{L}$. Тогда для $a \rightleftharpoons \pi_{L}^{\tau_{k}}$ имеем $\left(a u^{-1}\right)^{\tau-1}=1$, т.е. $\left(a u^{-1}\right)^{\tau}=$ $a u^{-1}, a u^{-1} \in A_{L^{\tau}}=A_{K}$. Тогда $v_{L}\left(a u^{-1}\right) \in v_{L}\left(A_{K}\right)=n Z$; но $v_{L}\left(u^{-1}\right)=0$, $v_{L}(a)=v_{L}\left(\pi_{L}^{\tau_{k}}\right)=v_{L}\left(\pi_{L}^{\tau^{k-1}+\tau^{k-1}+\cdots+\tau+1}\right)=k v_{L}\left(\pi_{L}\right)$. Так как $v_{L}\left(\pi_{L}\right) \cdot n Z-$ образуюшая групшы $Z / n Z$, то отсюда следует, что $n \mid k$ и, следовательно, $\tau^{k}=1$.

Итак, $\bar{\chi}: H \longrightarrow H^{-1}\left(H, U_{L}\right)$ - вложение; тогда и $\chi^{L}: H \longrightarrow U_{K}^{L} / N_{L / K}\left(U_{L}\right)$ является вложением. Коммутативная диаграмма на рис. 6 показьвает, что и гомоморфизм $\chi^{L^{\prime}}: H \longrightarrow U_{K}^{L^{\prime}} / N_{L^{\prime} / K}\left(U_{L}^{\prime}\right)=U_{K}^{L^{\prime}} / N_{L / K}\left(U_{L}\right) \leqslant U_{K} / N_{L / K}\left(U_{L}\right)$ является вложением. По следствию 5 имеем $r_{L / K}(\sigma)=\chi^{L^{\prime}}(\sigma) N_{L / K}\left(A_{L}\right)$ для $\sigma \in G=H=H_{K}^{L}=I_{K}^{L}$.

Для завершения доказательства осталось лишш заметить, что $N_{L / K}\left(A_{L}\right) \cap U_{L}=$ $N_{L / K}\left(U_{L}\right)$; тогда $U_{K} / N_{L / K}\left(U_{L}\right) \leqslant A_{K} / N_{L / K}\left(A_{L}\right)$ и $r_{L / K}$ - вложение.

Для завершения доказательства теоремы 1 рассмотрим случай произвольного (конечного) абелева расширения $M \geqslant K$. По предложению 3 найдется конечноенеразветвленное расширение $L \geqslant M$ такое, что эпиморфизм $d_{K}^{L}: G(L / K) \longrightarrow \mathbb{Z}_{L / K}$ 
расшепляется. Заметим теперь, что $L$ - абелево расширение $K$ и для расширения $L \geqslant K$ выполнено условие представления $L$ в виде композита циклических неразветвленных и вполне разветвленных расширений. Тогда по доказанному выше

$$
r_{L / K}: G(L / K) \longrightarrow A_{K} / N_{L / K}\left(A_{L}\right)
$$

- вложение, а по лемме $10 r_{L / M}: G(L / M) \longrightarrow A_{M} / N_{L / M}\left(A_{L}\right)$ - эпиморфизм (даже изоморфизм). Следствие 6 показьвает, что

$$
r_{M / K}: G(M / K) \longrightarrow A_{K} / N_{M / K}\left(A_{M}\right)
$$

является вложением. Теорема доказана.

Следующее условие назовем аксиомой $R$ : для любого вполне разветвленного циклического расширения $L \geqslant K$ простой степени вьполнено неравенство

$$
\left|H^{0}\left(G(L / K), A_{L}\right)\right| \leqslant[L: K]
$$

Пусть $d: G \longrightarrow \widehat{\mathbb{Z}}$ - эпиморфизм проконечных групп, $A$-непрерывный $G$-модуль, $v: A_{k} \longrightarrow \widehat{\mathbb{Z}}$ - гензелево нормирование (относительно $d$ ), и пусть выполнены аксиомы $U$ и $R$.

Тогда для пары $(d, v)$ справедлива теория полей классов, а именно справедлива

ТеОрема 2. Для любых конечных расширений $(k \leqslant) K \leqslant L$ maких, что $L-$ расиирение Галуа поля $K$, гомоморфизм взаимности

$$
r_{L / K}: G(L / K)^{\mathrm{ab}} \longrightarrow A_{K} / N_{L / K}\left(A_{L}\right)
$$

является изоморфизмом.

ДокАЗАТЕльство. Заметим, что для циклических расширений простой степени теорема справедлива. Действительно, если $L \geqslant K$ - неразветвленное расширение, то это следует из леммы 11 . Если $L \geqslant K$ - вполне разветвленное расширение, то по теореме 1

$$
r_{L / K}: G(L / K) \longrightarrow A_{K} / N_{L / K}\left(A_{L}\right)
$$

является вложением; следовательно,

$$
p=|G(L / K)| \leqslant\left|A_{K} / N_{L / K}\left(A_{L}\right)\right| \leqslant p
$$

влечет, что $\left|A_{K} / N_{L / K}\left(A_{L}\right)\right|=p$ и $r_{L / K}-$ изоморфизм. Используя диаграмму $(+)$ и индукцию по числу элементов групшы $G(L / K)$, легко установить справедливость теоремы 2 для всех разрешимых (или, что достаточно, нильпотентных) груп $G(L / K)$.

Для произвольной группы $G(L / K)$ ввиду теоремы 1 достаточно установить, что

$$
r_{L / K}: G(L / K) \longrightarrow A_{K} / N_{L / K}\left(A_{L}\right)
$$

является эпиморфизмом. 
Так как $N_{L / K}\left(A_{L}\right) \geqslant A_{K}^{[L: K]}$, то $A_{K} / N_{L / K}\left(A_{L}\right)$ - периодическая абелева группа. Пусть $p$ - произвольное простое число, $A_{p}-p$-примарная часть группы $A_{K} / N_{L / K}\left(A_{L}\right)$. Теперь достаточно доказать, что $A_{p} \leqslant r_{L / K}(G(L / K))$.

Пусть $P$ - силовская $p$-подгруппа группы $G(L / K), M \rightleftharpoons L^{P}$. Рассмотрим коммутативную диаграмму

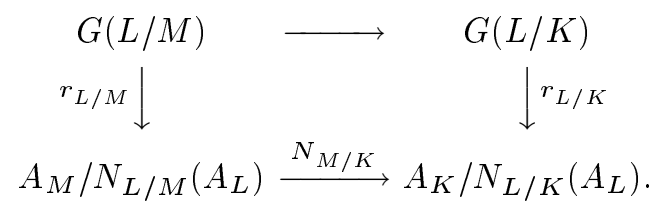

Так как $G(L / M) \simeq P$ - нильпотентная группа, то $r_{L / M}$ является эпиморфизмом и $A_{M} / N_{L / M}\left(A_{M}\right)=r_{L / M}(G(L / M))$ - абелева $p$-группа.

Включения $A_{K} \leqslant A_{M}$ и $N_{L / K}\left(A_{L}\right) \leqslant N_{M / K} N_{L / M}\left(A_{L}\right) \leqslant N_{L / M}\left(A_{L}\right)$ индуцируют гомоморфизм $i: A_{K} / N_{L / K}\left(A_{L}\right) \longrightarrow A_{M} / N_{L / M}\left(A_{L}\right)$ такой, что $N_{M / K} i=$ $[M: K]$. Так как $p \nmid[M: K]$, то $N_{M / K} i\left(A_{p}\right)=[M: K] A_{p}=A_{p}$ и

$$
A_{p} \leqslant N_{M / K}\left(A_{M} / N_{L / M}\left(A_{L}\right)\right)=N_{M / K} r_{L / M}(G(L / M))=r_{L / K}(G(L / K))
$$

что и требовалось.

Теорема 2 доказана.

Как показывает доказательство предложения (6.2) в [2], аксиомы $U$ и $R$ вместе эквивалентны следующей (слегка ослабленной) аксиоме полей классов:

для любого ииклического расширения $L \geqslant K$ имеет место неравенство

$$
\left|H^{0}\left(G(L / K), A_{L}\right)\right| \leqslant[L: K]
$$

$u\left|H^{-1}\left(G(L / K), A_{L}\right)\right|=1$, если $L$ неразветвлено над $K$.

Установим, наконец, что определенный вьше гомоморфизм взаимности $r_{L / K}$ совпадает с гомоморфизмом взаимности $r_{L / K}$, определенньм Нейкирхом в [2].

Пусть $L \geqslant K$ - расширение Галуа, $\widetilde{L}$ - наибольшее неразветвленное расширение $L$ (здесь мы отказываемся от рассмотрения только конечных расширений $k$; так, $\widetilde{L} \geqslant k$ - бесконечное расширение).

Представим $\widetilde{L}$ как объединение башни конечных расширений $(L \leqslant) L_{0} \leqslant L_{1} \leqslant$ $\cdots \leqslant L_{n} \leqslant \cdots$ такой, что $\left[L_{0}: L\right]=n_{L / K}$ (число $n_{L / K}-$ из предложения 3 ), $\widetilde{L}=\bigcup_{n \in \omega} L_{n}$.

Для любого $\sigma \in G(L / K)$ в $\operatorname{Frob}\left(L_{n} / K\right)$ имеется подъем $\sigma_{n}$ и $\sigma_{n+1} \uparrow L_{n}=\sigma_{n}$ (по лемме 2). Тогда сушествует элемент $\bar{\sigma} \in G(\widetilde{L} / K)=\lim _{n \in \omega} G\left(L_{n} / K\right)$ такой, что $\bar{\sigma} \uparrow L_{n}=\sigma_{n}$ для всех $n \in \omega$. Тогда $\bar{\sigma}$ является фробениусовым элементом $(\bar{\sigma} \in$ $\operatorname{Frob}(\widetilde{L} / K))$ в смысле Нейкирха, так как по построению имеет место соотношение $d_{K}(\bar{\sigma})=\Phi^{\partial_{K}^{L}(\sigma)}$, и $\bar{\sigma} \uparrow L=\sigma$, т.е. $\bar{\sigma}$ является подъемом $\sigma$ (в смысле Нейкирха). Если $\pi$-простой элемент $A_{\widetilde{L}}^{\bar{\sigma}}=A_{L_{0}}^{\sigma_{0}}$, то $r_{L / K}(\sigma)=N_{L_{0}^{\sigma_{0} / K}}(\pi) N_{L / K}\left(A_{L}\right)$ совпадает со значением $N_{\widetilde{L}^{\bar{\sigma}} / K}(\pi) N_{L / K}\left(A_{L}\right)$ гомоморфизма взаимности на $\sigma$, определенным Нейкирхом в [2; предложение (5.6)]. 


\section{Зак лючение}

Предложенная в статье теория может быть обобшена (расширена) в следуюших двух направлениях.

I. Следуя идее упражнения 2 из $[2 ;$ гл. IV,$\S 6]$, возможен следующий вариант абстрактной теории полей классов для про-П-групп, где П- произвольное множество простых чисел.

Для простого $p$ пусть $\mathbb{Z}_{p} \rightleftharpoons \lim _{n \in \omega} \mathbb{Z} / p \mathbb{Z}$ (группа $\mathbb{Z}_{p}$ изоморфна аддитивной группе целых $p$-адических чисел); $\mathbb{Z}_{\Pi} \rightleftharpoons \prod_{p \in \Pi} \mathbb{Z}_{p}$.

Заметим, что если П совпадает с множеством $\mathscr{P}$ всех простых чисел, то $\mathbb{Z} \mathscr{P} \simeq \widehat{\mathbb{Z}}$.

Пусть $G$ - про-П-группа, $d: G \longrightarrow \mathbb{Z}_{\Pi}-$ эпиморфизм.

Большинство понятий (группа инерции, степень инерции, индекс ветвления, неразветвленное и вполне разветвленное расширение и др.) вводятся так же, как в основном тексте статьи.

Сушественным является правильное определение понятия фробениусова элемента.

Натуральное число $n>0$ называется П-числом, если все простые делители $n$ принадлежат П; П-содержанием $n_{\Pi}$ натурального числа $n>0$ назьвается наибольший П-делитель числа $n\left(0_{\Pi} \rightleftharpoons 0\right)$.

Если $L \geqslant K$ - расширение Галуа (и $[L: k]<\omega$ ), то элемент $\sigma \in G(L / K)$ $\left(\rightleftharpoons G_{K} / G_{L}\right)$ называется әлементом Фробениуса $(\sigma \in \operatorname{Frob}(L / K))$, если

1) $\langle\sigma\rangle \cap I_{K}^{L}=\langle 1\rangle$,

2) $|\sigma| \cdot\left(\partial_{K}^{L} \sigma\right)_{\Pi}=f_{L / K}$.

Здесь $\partial_{K}^{L} \sigma$ имеет тот же смысл, что и в основном тексте статьи, если зафиксировать образуюшую $\Phi$ групшы $\mathbb{Z}_{\Pi}$.

Важным изменением является следующий аналог свойства 3 фробениусовых элементов.

$3_{\Pi}$. Если $\sigma \in \operatorname{Frob}(L / K), m>0$ таково, что $m \partial_{K}^{L} \sigma \leqslant f_{L / K}$ и $m_{\Pi}$ делит $|\sigma|$, то $\sigma^{m} \in \operatorname{Frob}(L / K)$ и $\partial_{K}^{L} \sigma^{m}=m \partial_{K}^{L} \sigma\left(\left|\sigma^{m}\right|=|\sigma| \cdot m_{\Pi}^{-1}\right)$.

Лемма 4 для конечной П-групшы $G$ справедлива в следуюшей формулировке.

ЛЕмма 4 П. Если $g \in G,\langle g\rangle \cap H=\langle 1\rangle$, и порядок $m \rightleftharpoons|g|$ әлемента $g$ делится на $\Omega(H)_{\Pi}$, то для любого $h \in H$ порядок әлемента gh равен $m u$ $\langle g h\rangle \cap H=\langle 1\rangle$.

Для справедливости предложения 3 в качестве числа $n_{L / K}$ нужно взять П-содержание числа, указанного в доказательстве этого предложения в основном тексте статьи.

Определение понятия гензелева нормирования $v: A_{K} \longrightarrow \mathbb{Z}_{\Pi}$ (по отношению к $\left.d: G \longrightarrow \mathbb{Z}_{\Pi}\right)$ модифицируется естественным образом.

Далее все основные конструкции (отображения Нейкирха, Хазевинкеля и взаимности) и их свойства остаются справедливыми, включая последнюю теорему об изоморфизме $G(L / K)^{\mathrm{ab}} \stackrel{\sim}{\longrightarrow} A_{K} / N_{L / K}\left(A_{L}\right)$ при выполнении аксиомы полей классов.

II. Возможно следующее обобщение понятия гензелева нормирования (по отношению к эпиморфизму $d: G \longrightarrow \widehat{\mathbb{Z}})$. 
“Гензелевым" нормированием (мультипликативного непрерывного) $G$-модуля $A$ (по отношению к эпиморфизму $d$ ) называется гомоморфизм

$$
v: A_{k} \longrightarrow \widehat{\mathbb{Z}}
$$

такой, что для $Z \rightleftharpoons v\left(A_{k}\right) \leqslant \widehat{\mathbb{Z}}$ вьполнены условия:

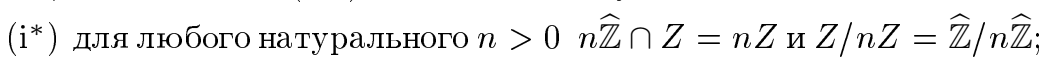

(ii) $v\left(N_{K / k}\left(A_{K}\right)\right)=f_{K} Z$ для любого $K$ такого, что $[K: k]<\omega$.

Условие $\left(i^{*}\right)$ эквивалентно совокупности двух условий:

(i') $Z$ сервантна в $\widehat{\mathbb{Z}}$;

$\left(\mathrm{i}^{\prime \prime}\right) \widehat{\mathbb{Z}} / Z$ - делимая групша.

ЗАмЕчАниЕ 11. Отличие “гензелева" нормирования от гензелева нормирования состоит в следуюшем: не предполагается, что $Z$ содержит (какую-нибудь) образующую группы $\widehat{\mathbb{Z}}$.

Укажем, как нужно модифицировать определение отображения Нейкирха в случае "гензелева" нормирования.

Пусть $K \geqslant k$ - конечное расширение, $n>0 ; n$-nростым элементом $A_{K}$ называется любой элемент $\pi \in A_{K}$ такой, что

$$
v_{K}(\pi)+n \widehat{\mathbb{Z}}=\Phi+n \widehat{\mathbb{Z}} .
$$

Пусть $L \geqslant K$ - расширение Галуа $([L: k]<\omega)$; отображение Нейкирха

$$
\nu_{L / K}^{*}: \operatorname{Frob}(L / K) \longrightarrow A_{K} / N_{L / K}\left(A_{L}\right)
$$

определим так: пусть $\sigma \in \operatorname{Frob}(L / K), \Sigma \rightleftharpoons L^{\sigma}, \pi_{\Sigma}-[L: K]$-простой элемент $A_{\Sigma}$; тогда

$$
\nu_{L / K}^{*}(\sigma) \rightleftharpoons N_{\Sigma / K}\left(\pi_{\Sigma}\right) \cdot N_{L / K}\left(A_{L}\right) .
$$

Нетрудно проверить корректность этого определения.

Заметим, что в основном тексте статьи отображение Нейкирха $\nu_{L} / K$ отображает $\operatorname{Frob}(L / K)$ в $A_{K} / N_{L / K}\left(U_{L}\right)$ (а не в $A_{K} / N_{L / K}\left(A_{L}\right)$, как определял сам Нейкирх в [2]).

Это отображение $\nu_{L / K}^{*}$ позволяет определить гомоморфизм взаимности $r_{L / K}$ со всеми нужными свойствами.

III. Обобщения из I и II безусловно могут быть естественно реализованы совместно.

\section{Список литературы}

1. Neukirch J. Class field theory. Berlin: Springer-Verlag, 1986.

2. Neukirch J. Algebraic number theory. Chapter IV: Abstract class field theory. Berlin: Springer-Verlag, 1999.

3. Ивасава К. Локальная теория полей классов. М.: Мир, 1983.

4. Waterhouse W. C. Profinite groups are Galois groups // Proc. Amer. Math. Soc. 1974. V. 42. № 2. P. 639-640.

5. Ершов Ю. Л. Алгоритмические проблемы в теории полей (положительные аспекты) // Справочная книга по математической логике. Часть 3: Теория рекурсии. М.: Наука, 1982. C. 269-353. 